\title{
Estimation of individual growth trajectories when repeated measures are missing
}

\author{
Brooks, Mollie Elizabeth; Clements, Christopher; Pemberton, Josephine; Ozgul, Arpat
}

\section{Published in:}

American Naturalist

Link to article, DOI:

$10.1086 / 692797$

Publication date:

2017

Document Version

Peer reviewed version

Link back to DTU Orbit

Citation (APA):

Brooks, M. E., Clements, C., Pemberton, J., \& Ozgul, A. (2017). Estimation of individual growth trajectories when repeated measures are missing. American Naturalist, 190(3), 377-388. https://doi.org/10.1086/692797

\section{General rights}

Copyright and moral rights for the publications made accessible in the public portal are retained by the authors and/or other copyright owners and it is a condition of accessing publications that users recognise and abide by the legal requirements associated with these rights.

- Users may download and print one copy of any publication from the public portal for the purpose of private study or research.

- You may not further distribute the material or use it for any profit-making activity or commercial gain

- You may freely distribute the URL identifying the publication in the public portal 


\section{Estimation of individual growth trajectories when repeated measures are missing}

Mollie E. Brooks ${ }^{*}$ 1,2, Christopher Clements ${ }^{1}$, Josephine Pemberton ${ }^{3}$, and Arpat Ozgul 1

${ }^{1}$ Department of Evolutionary Biology and Environmental Studies, University of Zurich, Winterthurerstrasse 190, 8057 Zurich, Switzerland

${ }^{2}$ National Institute of Aquatic Resources, Technical University of Denmark, Charlottenlund Slot, 2920 Charlottenlund, Denmark

${ }^{3}$ Institute of Evolutionary Biology, University of Edinburgh, Edinburgh EH9 3JT, United Kingdom

*Corresponding author: MollieEBrooks@gmail.com

Keywords: Template Model Builder, Soay sheep, individual quality, reproductive costs, state-space model, time-series

\section{Author-Supplied PDFs in the expanded online edition:}

Online Appendix S1 - Model with first mass covariates

Online Appendix S2 - The most parsimonious model

Online Appendix S3 - Extended results of simulation study

Online Appendix S4 - Model selection results

Dryad data: http://dx.doi.org/10.5061/dryad.r6j80 


\begin{abstract}
Individuals in a population vary in their growth due to hidden and observed factors such as age, genetics, environment, disease, and carryover effects from past environments. Because size affects fitness, growth trajectories scale up to affect population dynamics. However, it can be difficult to estimate growth in data from wild populations with missing observations and observation error. Previous work has shown that linear mixed models (LMMs) underestimate hidden individual heterogeneity when over $25 \%$ of repeated measures are missing. Here we demonstrate a flexible and robust way to model growth trajectories.

We show that state-space models (SSMs), fit using R package growmod, are far less biased than LMMs when fit to simulated datasets with missing repeated measures and observation error. This method is much faster than MCMC methods, allowing more models to be tested in a shorter time. For the scenarios we simulated, SSMs gave estimates with little bias when up to $87.5 \%$ of repeated measures were missing.

We use this method to quantify growth of Soay sheep, using data from a long-term mark-recapture study, and demonstrate that growth decreased with age, population density, weather conditions, and when individuals are reproductive. The method improves our ability to quantify how growth varies among individuals in response to their attributes and the environments they experience, with particular relevance for wild populations.
\end{abstract}




\section{Introduction}

Individuals in a population vary in their growth rates due to factors such as age, size, environment, behavioral traits, quality, genetics, disease, and carryover effects (Arnold 1981; Weiner 1985; Coleman and Wilson 1998; Pfister and Stevens 2002; Morgan et al. 2003; Sih et al. 2004; Wilson and Nussey 2009; Nussey et al. 2011; O'Connor et al. 2014).. For example, a long-term mark-recapture study of Soay sheep has revealed that individual growth trajectories are influenced by senescence, population density, weather, genetics, and maternal effects (Clutton-Brock and Pemberton 2004; Ozgul et al. 2009). Because individual size has consequences for individual fitness and among-individual interactions, growth patterns scale up to affect population demography and community dynamics (Emmerson and Raffaelli 2004; Pfister and Wang 2005; de Valpine et al. 2014). Thus quantifying individual growth trajectories is of fundamental interest in ecology.

Structured population models most commonly account for variation among individuals that is attributable to observed covariates such as size, age, or developmental stage. However, after accounting for covariates, individuals may additionally differ in their growth trajectories due to hidden factors such as genetics, maternal effects, parasite load, or other aspects of individual quality (Brienen et al. 2006; Wilson and Nussey 2009; Kendall et al. 2011). Whether the cause is hidden or observed, individual growth trajectories are influenced by a mixture of persistent (e.g., genotype, personality) and transient (e.g., parasite load, resource availability) heterogeneity. These are also referred to as "constant", "fixed", or "static" versus "labile" or "dynamic" (Tuljapurkar et al. 2009; Vindenes and Langangen 2015; Bonnet and Postma 2016; Childs et al. 2016). Here we use the terms "persistent" and "transient" (Kendall et al. 2011; Webber and Thorson 2015).

Persistent heterogeneity in individual growth is especially important because fast growing individuals quickly reach reproductive sizes, and thus increase the population 
growth rate (Pfister and Wang 2005; Zuidema et al. 2009; de Valpine et al. 2014). Or, it can change the amplitude and frequency of population cycles (Lindström and Kokko 2002; Wearing et al. 2004). Heterogeneity also plays an important role in eco-evolutionary dynamics, leading recent work to argue that it is sometimes necessary to include persistent individual heterogeneity in population models (Hedrick et al. 2014; Chevin 2015; Vindenes and Langangen 2015; Childs et al. 2016). However, doing so will require adequate methods of estimation.

Methods of quantifying growth patterns can be divided into autoregressive and sizeat-age models. Autoregressive models use an individual's current size as a predictor of its future size. Treating size as a Markov process, as is commonly done for structured population models, is a first-order autoregressive (AR(1)) model, e.g., the Ford-Walford growth model (Ford 1933; Walford 1946; Easterling et al. 2000; Thorson et al. 2015b). Sizeat-age models, on the other hand, estimate size as a function of age. Simple autoregressive models can be solved for their equivalent size-at-age models, (Walford 1946; Bertalanffy 1957), but this becomes difficult or impossible for more complex models such as those used to estimate individual growth trajectories. Therefore this distinction is not always applicable, but we use it here to describe general strengths and weaknesses of autoregressive and size-at-age models.

Recent work has quantified individual growth trajectories based on size-at-age models including the logistic, von Bertalanffy, and Monomolecular (Sainsbury 1980; Cheng and Kuk 2002; English et al. 2011; Sofaer et al. 2013; Huchard et al. 2014; Vincenzi et al. 2014; Webber and Thorson 2015). These methods have the strength of being able to use repeated observations taken at irregular time intervals. For example, in the long-term study of Soay sheep cited above, weights are observed each summer, but only $50 \%$ of individuals are captured, creating gaps in the time-series - gaps which would not cause an issue for 
size-at-age models. This type of missing repeated measure is common in surveys of wild populations where recapture rates are imperfect. The simplicity of size-at-age models is appealing because the shape is controlled by just a few parameters in which individuals could vary; however, this limits their flexibility for including temporally varying predictors and carrying over effects from previous environments because the parameters are fixed throughout an individual's ontogeny (although see (Szalai et al. 2003)). All but two (Cheng and Kuk 2002; Webber and Thorson 2015) of the size-at-age models cited above are at risk of misestimating persistent individual heterogeneity because they ignore transient types of temporal autocorrelation in body size (Brienen et al. 2006; Hamel et al. 2012; Brooks et al. 2013). Quantitative geneticists recommend modeling age-dependent traits, such as size, using process models that also account for plastic environmental effects and temporal autocorrelation of the trait (Pletcher and Geyer 1999; Stinchcombe and Kirkpatrick 2012; Hadfield et al. 2013). The need to include plasticity in growth was also recently highlighted in fisheries, where management targets are highly sensitive to changes in growth parameters (Thorson et al. 2015a; Lorenzen 2016).

An alternative to size-at-age models are AR(1) growth models, which use an individual's previous size to predict its size at the next time point (Walford 1946). AR(1) models automatically account for the transient component of individual heterogeneity and other causes of temporal autocorrelation in the time series. They are more flexible than size-at-age models and consequently effects of temporally varying predictors, such as population density or weather, can be included in the growth trajectory (e.g., Ozgul et al. 2014). For example, to investigate how climate change could affect body size and population density of Soay sheep, Simmons and Coulson (2015) modeled yearly changes in individual size as an AR(1) process that varies with weather and population density. Because of the autoregressive nature of AR(1) models, some of the effects of temporally varying predictors 
carry over from one age to the next. AR(1) models can estimate persistent heterogeneity due to unobserved variables by including a random intercept for each individual (Cam et al. 2012; Hamel et al. 2012). However, a weakness of AR(1) models is that the previous mass is needed to predict the following mass; this means that observations for which the previous observation is missing must be discarded (as was done in the sheep growth study cited above), or missing observations must be imputed (Nakagawa and Freckleton 2008). Discarding data reduces statistical power. Imputation is complicated (Horton and Kleinman 2007) and often requires making assumptions about the growth pattern which is tautological when the growth pattern is what is being determined. Another weakness of AR(1) models is that they are sensitive to observation error in the response variable unless it is accounted for; otherwise, they contain temporally autocorrelated noise because the model for one imperfect observation contains the error of both that observation and the observation before it (for more detail see Dennis et al. 2006).

Hamel et al. (2012) conducted a simulation study to compare the ability of AR(1) and size-at-age models to estimate persistent individual heterogeneity and temporal autocorrelation in demographic datasets with missing values and no imputation. They demonstrated that for datasets with many missing repeated measures and high individual heterogeneity, AR(1) models underestimate individual heterogeneity and with many missing repeated measures, both types of models produce biased estimates of temporal autocorrelation. As a result, they recommend against using either type of model when more than $25 \%$ of repeated measures are missing. However, these have been the only readily available methods, so researchers still try to use them to estimate individual heterogeneity in growth for datasets with more missing repeated measures than the recommended limit (Plard et al. 2015). 
One way to take advantage of the flexibility of the AR(1) approach while still using measurements for which the previous measurement is missing is to model growth in a state-space framework (Clark et al. 2007; Shelton and Mangel 2012; Sigourney et al. 2012; Shelton et al. 2013). State-space methods model time series data using a process model for the true latent state (size in the case of a growth process) and another model for drawing observations from that process (Kitagawa 1987; de Valpine and Hastings 2002). Thus, the process is estimated even at points when observations are not taken because points are linked in series by either a difference equation or a differential equation. Taking a statespace approach has several benefits. It can account for observation errors and temporal autocorrelation, both of which can bias estimates (Carroll et al. 2006; Hamel et al. 2012). Also, it automatically produces interpolated values for missing observations - values needed for estimating the relationship between individual size and other demographic rates needed for size-structured population models and some capture-recapture analyses. A weakness of the state-space approach is that it requires covariates from the beginning of an individual's life to predict the process. So this approach is only applicable to individuals born after covariate (e.g., population density) observation began whereas simpler AR(1) models can use observations of any individual. Another weakness of state-space models is that parameter estimation in non-trivial models has traditionally required either MCMC sampling or expectation maximization methods, which are too slow to allow for extensive model selection.

Here, we circumvent this issue by developing a new method, which fits state-space models via maximum likelihood estimation to vastly speed up model fitting, and show (with simulated data) that state-space models avoid the biases of simpler AR(1) models when repeated measures are missing and when observations contain errors. We use this method to do extensive model selection and find the most parsimonious model to account for 
factors that influence variation in growth trajectories for a long-term mark-recapture study of Soay sheep.

\section{Methods}

\section{State-space growth model}

Our growth model treated an individual's true size, $m_{i, t}$ for individual $i$ in year $t$, as a latent variable. This allowed missing sizes to be filled in by the process model, which links consecutive values of the latent variable. We modeled size on the log scale to ensure that it stays positive on the natural scale, but this won't be required for all study systems.

Modeling on the log scale has the additional consequence that effects are multiplicative on the natural scale. Our model allows growth (i.e., changes in size) to be positive or negative throughout ontogeny, as is common for leaf area in plants (Salguero-Gómez and Casper 2010), weight in animals (e.g., Nussey et al. 2011), and occasionally body length in animals (e.g., Wikelski and Thom 2000). Thus we incorporate multiple biological processes (e.g., ontogenetic growth, changes in condition, and senescence) into one model.

We assumed that size in the first time point of life was drawn from a normal distribution with the same mean and variance for all individuals $m_{i, t 0_{i}} \sim N\left(\mu_{0}, \sigma_{0}^{2}\right)$, given birth year $t O_{i}$ for individual $i$. Then, sizes were projected forward for each time step of each individual's life using a mixed effect model structure that included individual and environmental fixed effect covariates and random effects to quantify deviations from the average growth trajectory. The AR(1) process model can be represented as $m_{i, t}=m_{i, t-1} M_{i, t} \eta+X_{i, t} \beta+z_{i}+c_{t 0_{i}}+v_{t}+\varepsilon_{i, t}$ eqn 1 where $X$ and $M$ are design matrices specifying the fixed effect formula including autoregressive components; $\beta$ and $\eta$ are the corresponding vectors of coefficients. $\varepsilon_{i, t}$ is Gaussian distributed process error, $\varepsilon_{i, t} \sim N\left(0, \sigma_{\text {process }}^{2}\right)$. We included random effects of 
individual, $z_{i} \sim N\left(0, \sigma_{\text {individual }}^{2}\right)$; birth cohort, $c_{t 0_{i}} \sim N\left(0, \sigma_{\text {cohort }}^{2}\right)$; and time, $v_{t} \sim N\left(0, \sigma_{\text {time }}^{2}\right)$ on the intercept. These are Gaussian distributed random deviations from the average growth trajectory. In this formulation, the random effects of individual and cohort are persistent throughout an individual's life and affect the entire ontogeny; thus they ignore other types of heterogeneity that do not persist throughout the entire ontogeny, such as compensatory growth. In models with age as a continuous variable, we also considered a random slope of age for each individual and estimated its correlation with the individual intercept. This slope could also capture compensatory growth. We did not consider a random slope of age for cohorts, so we ignore compensatory growth of that type (but see Hamel et al. 2016). Our state-space model assumed that observed sizes, $o$, contain Gaussian distributed error around the true latent size such that $o_{i, t} \sim \mathrm{N}\left(m_{i, t}, \sigma_{\text {observation }}^{2}\right)$ $o_{i, t} \sim \mathrm{N}\left(m_{i, t}, \sigma_{\text {observation }}^{2}\right)$. Thus, the joint likelihood of this state-space model contains the likelihoods of each of the following components: the latent initial sizes, $m_{i, t 0_{i}}$; the process errors, $\varepsilon_{i, t} ;$ the observation errors, $o_{i, t}-m_{i, t}$; the random deviations for each individual, $z_{i}$; the random deviations for each birth cohort, $c_{t 0_{i}}$; and the random deviations for each time step, $v_{t}$.

Code for fitting this model in $\mathrm{R}$ is available in a package called growmod, which can be downloaded from GitHub (https://github.com/mebrooks/growmod). The package can fit all models described in this text and includes functions for extracting estimated coefficients with standard error estimates and predictions. More details can be found in the section Model fitting using TMB below.

\section{Simulation study comparing LMMs and state-space models}

A linear mixed model (LMM) framework is commonly used to fit growth as an AR(1) process. This differs from our state-space approach because observations for which the 
previous observation is missing are omitted, and observation error is ignored. It is possible to estimate observation error using LMMs by making assumptions about the correlation structure (Dennis et al. 2006), but their ability to concurrently estimate random effects of time and individual has not been established. Consequently, the LMMs discussed here ignore observation error. To test for differences in bias, we applied LMMs and our statespace model to simulated data described below. We simulated data from a model that included previous mass, a quadratic effect of age, an interaction of age and previous mass, and random intercepts for each individual and year. Each simulated data set contained 29 years of observations of 1500 individuals with random birth years and longevities randomly sampled with replacement from those observed in the case study described below. In the simulations, we varied the recapture rate $(0.05,0.125,0.25,0.5,0.75$, and 1.0$)$ and the coefficient of variation of observation error $\left(\mathrm{cv}_{\mathrm{obs}}=0,0.006,0.012\right.$, and 0.018$)$. Simulated observations did not allow the same individual to be recaptured at the same time point, which is a sub-optimal design for estimating observation error (Dennis et al. 2010). We did 100 simulations for each combination of recapture probability and $\mathrm{cv}_{\text {obs. }}$. LMMs were fit using restricted maximum likelihood via the lmer function of lme4 in the R statistical computing environment (Bates et al. 2015a; R Core Development Team 2016). Details of the simulations can be found in a vignette using the R command vignette("sim_growmod").

\section{Soay sheep case study}

We modeled the growth in body mass of Soay sheep (Ovis aries) using a long-term data set of marked individuals from the Island of Hirta in the St. Kilda archipelago of Scotland, UK. In this long-term study, beginning in 1985, sheep were captured and weighed every August, with about 0.5 annual recapture probability (Clutton-Brock and Pemberton 2004). We assumed that recapture success was random with a constant probability given 
that an individual was alive. Reproductive status was recorded based on observed birth or suckling (Childs et al. 2011) which can vary from year-to-year within an individual and is not synonymous with sexual maturity. This only includes live births and excludes individuals that died before giving birth or those that gave birth to dead fetuses; it also does not differentiate between lambs that survived to weaning from those that died. The population density was measured during island-wide counts since 1986; here we use the local population density (i.e., the village population) (Ozgul et al. 2009; Tavecchia et al. 2009; Childs et al. 2011). Lambs are born in April and May and can reproduce in the following spring, but the proportion reproducing in their first year varies from 6 to $80 \%$ depending on the environment (Clutton-Brock and Pemberton 2004). Previous work on this population has shown that growth varies by life stage and age, and that growth is reduced in years with high population density and for lambs that reproduce in their first year of life (Ozgul et al. 2009; Childs et al. 2011; Nussey et al. 2011). Previous work also found effects on lamb growth from the North Atlantic Oscillation (NAO) and NAO interacting with population density as well as effects on adult growth from NAO, reproductive effort, and their interaction, all close to statistical significance (Ozgul et al. 2009). Birth mass in spring is also affected by maternal stage (yearling versus adult), maternal mass, population density, and being born as a twin (Childs et al. 2011). Here, in the main text we ignore predictors of mass in the first August of life because the necessary predictors were only available for a subset of individuals. In online appendix S1, we demonstrate that this does not affect our general results.

We restricted our analyses to individuals born after 1986, the period when necessary predictors were available, and used data up to 2014. For simplicity, we focused our study on females, but sex could easily be included as a covariate. Using these criteria gave us 4038 masses of 1560 unique females with a median age of 3 years, observed across 29 years. 
Information on maternal age and maternal mass in the year of birth and being born as a twin were available for 951 individuals with 2566 mass observations. Data are deposited in the Dryad Digital Repository: http://dx.doi.org/10.5061/dryad.r6j80 (Brooks et al. 2017).

There were 226 repeated observations of 112 individuals which we used to estimate the distribution of observation errors (Carroll et al. 2006). Assuming that individual $i$ was measured $k_{i}>1$ times in August of the same year, then the variance of observations around the true masses could be estimated as

$$
\sigma_{\text {observation }}^{2}=\frac{\sum_{i=1}^{n} \sum_{j=1}^{k_{i}}\left(o_{i, j}-\bar{o}_{i}\right)}{\sum_{i=1}^{n}\left(k_{i}-1\right)}
$$

For simplicity, we omitted year $(t)$ from the notation in eqn 2 because the relevant repeated measurements of an individual (i) occur within the same year. This estimate of observation error also included error due to different dates of observation within the month of August. It is possible to estimate observation and process error simultaneously within a state-space model, but we chose to take advantage of the repeated measurements to speed up model fitting. We verified the robustness of this estimate by refitting the most parsimonious model while simultaneously estimating observation error.

\section{Sheep growth covariates and model selection}

We performed model selection on individual and environmental covariates in addition to the random effects using Akaike information criterion, AIC. In the AIC calculations, we counted each estimated variance term, as one parameter because our goal was to do inference at the population, rather than individual level (Bolker 2015).

Alternatively, if the goal was to do inference at the individual level, then each individual's deviation from the average intercept could count as, at most, one parameter (Vaida and Blanchard 2005). For this analysis, the maximum number of parameters was small relative to the number of observations so we did not perform any small-sample bias correction, but 
analyses of smaller data sets may need to do so (Cavanaugh and Shumway 1997; Bengtsson and Cavanaugh 2006).

Individual covariates included age, lamb status (whether or not they were born in year $t$-1), mass in year $t$-1, and reproductive status in year $t$ (whether or not a female gave birth to a live fetus in the current year). Recent work on this population has treated age in various ways (Ozgul et al. 2009; Childs et al. 2011; Nussey et al. 2011); so, we tested versions that included age as linear, quadratic, a factor, or in stage classes as defined by Ozgul et al. (2009). The factor of age was defined with one level per age, except ages over twelve, which were combined into one level. We also included lamb status as a factor in models with age as a continuous variable or with no other effect of age to model the discontinuous difference between lambs and ewes; i.e., the lamb effect is essentially a discontinuity in the age effect.

Environmental covariates included population density in year $t-1$ and two versions of the North Atlantic Oscillation index (NAO): winter NAO (WNAO) was averaged over December (in year $t$-1) to March (in year $t$ ); summer NAO (SNAO) was averaged over May through August (in year $t$ ). We did not include both versions of NAO in the same model due to their high correlation. Population density was scaled to have mean zero and unit variance. We considered interactions supported by earlier work including lamb status and population density; lamb status and NAO; lamb status and reproductive status; lamb status, NAO, and population density; lamb status and mass; mass and NAO; reproductive status and NAO; and reproductive status and age (Ozgul et al. 2009; Childs et al. 2011).

To select the form for the age effect, we limited our comparison to models containing random intercepts for individuals, birth cohorts, and years, but compared all combinations of covariates for each form of the age effect. Then, given that form of the age effect, we tested all combinations of covariates in models with additional random slopes on 
age for individuals. We estimated individual slopes and intercepts as multivariate normal with correlation. We also did model selection on versions without cohort effects. Finally, we compared the most parsimonious model for the sheep to a LMM of the same form.

To make effect sizes interpretable, we calculated predictions on the natural scale by back-transforming them. Assuming that $m$, the predicted size on the log scale, is normally

distributed with total variance $\sigma_{\text {total }}^{2}$, directly implies that the back-transformed values are $\log$-normally distributed with mean $\exp \left(m+0.5 \sigma_{\text {total }}^{2}\right)$.

With the most parsimonious model, we examined the estimated individual random deviations from the average growth intercept and quantified their correlation with maximum mass, longevity, and lifetime reproduction. We also checked for a relationship with reproduction in the first year of life. We tested the statistical significance of each relationship using likelihood-ratio tests performed on linear mixed models with a random effect of birth cohort.

\section{Model fitting using TMB}

We fit the state-space models using TMB, an R package for conducting maximum likelihood estimation of non-linear models containing continuous random effects (Kristensen et al. 2016). Non-technical readers can safely skip the remainder of the Methods section. In TMB, using a superset of $\mathrm{C}++$, the user defines the joint negative log-likelihood of their model. The difficulty of this first step is comparable to writing a model in Bayesian programs that use Gibbs sampling such as NIMBLE, WinBUGS, or JAGS. Then, TMB calculates the marginal negative log-likelihood by integrating out the latent variables $\left(m_{i, t}, z_{i}, c_{i, t 0_{i}}, v_{t}\right)$ using the Laplace approximation and calculates the gradient of the likelihood using automatic differentiation. With the marginal negative log-likelihood and the gradient, the model can be fit using any optimizer in R such as optim or nlminb. Maximizing the 
marginal likelihood is also known as the empirical Bayes method (de Valpine 2009;

Vincenzi et al. 2014).

Unlike other methods for fitting non-trivial state-space models, the algorithm used by TMB does not require prior distributions for parameters and is less sensitive to initial values (Bolker et al. 2013). Thus, we initialized parameters to uninformed values and initialized the latent masses, $m$, based on observed masses, $o$, filling in missing observations with the average mass. All fixed effect coefficients $(\eta, \beta)$ and random deviations $(z, c, v)$ were initialized to zero, with the exception of the autoregressive intercept, the first element of $\eta$, which was initialized to 0.1 because we assumed the best estimate would be positive. Variance parameters $\left(\sigma_{\text {process }}^{2}, \sigma_{\text {individual }}^{2}, \sigma_{\text {cohort }}^{2}, \sigma_{\text {year }}^{2}\right)$ were initialized to one and bounded to be positive by estimating them on the log scale. We used the same initial values for all model versions in model selection. For the most parsimonious model, we subsequently confirmed that results were consistent when initializing parameters to other starting values.

All state-space models were fit in R version 3.2.3 using TMB version 1.7.0 (Kristensen et al. 2016; R Core Development Team 2016). We did not compare TMB to MCMC sampling methods (e.g., OpenBUGS, JAGS) because previous studies have shown that TMB is typically 100 to 500 times faster than MCMC sampling (Pedersen et al. 2011; Bolker et al. 2013; Kristensen et al. 2016). We recorded the amount of time it took to fit our models on a laptop computer (MacBook with $2.7 \mathrm{GHz}$ Intel Core i7 processor, 8 cores, and 16 GB memory), but timing will vary with hardware. We ran separate models in parallel on 7 cores using the parLapply function (R Core Development Team 2016). We calculated 
confidence intervals for parameters using standard errors estimated by TMB using the delta method.

\section{Results}

\section{Simulation study comparing LMMs and state-space models}

The state-space model was generally less biased than the LMM (Figure 1). LMMs underestimated individual heterogeneity for lower recapture probabilities, e.g., -68\% mean (-100\% median) bias for 0.25 recapture rate and no observation error, whereas the statespace models had less than $1 \%$ mean (and median) bias. With low recapture rates, the LMM tended to underestimate the (age specific) intercept and overestimate the (age specific) slope on previous size ( $M \eta$ in eqn 1 ). For data sets with 0.125 recapture rate and no observation error, state-space models estimated fixed effects, as well as temporal and individual heterogeneity with mean bias at or below the order of $10^{-3}$, which translates to a percent error of 0.6 up to 5000 and only $3 \%$ mean (2\% median) bias for individual heterogeneity (detailed results in appendix S3). For data sets with 0.05 recapture rate and no observation error, LMMs could not be estimated while SSMs could be estimated for 85 out of 100 data sets but with considerable bias as detailed in online appendix S3. Observation error caused LMM estimates to be biased in the opposite direction from the biases caused by low recapture rates (Figure 1).

Given the recapture rate $(0.5)$ and $\mathrm{cv}_{\mathrm{obs}}(0.012)$ from the sheep data, estimates from LMMs had average bias below the order of 0.1 except the age effect. On average, LMMs underestimated individual heterogeneity by $58 \%$ ( -0.03 bias), and overestimated process error by $57 \%$ ( 0.03 bias). LMMs also had bias on the order of $10^{-3}$ to $10^{-1}$ (ranging from $>100 \%$ up to $80,000 \%$ ) for all fixed effect terms containing age (appendix S3). For the same simulated data, state-space models were very precise except that they overestimated the 
effect of age on the intercept by $34 \%\left(10^{-3}\right.$ bias $)$ and overestimated the effect of age on the slope of previous size by $800 \%\left(<10^{-4}\right.$ bias $)$.

\section{Soay sheep study results}

Based on model selection, a continuous quadratic effect of age was more parsimonious than including age as either a factor or a stage (Table 1). Given the quadratic age effect, the highest ranked random effects structure contained random intercepts for individuals, years, and birth cohorts; and a random slope on the age effect for each individual. However, the correlation of individual random slopes and intercepts was -1 , which is on the boundary of parameter space and indicates that the model was degenerate (Bates et al. 2015b); so we dropped the random slope from what we consider to be the most parsimonious model. See online appendix S4 for more model selection details. Given the set of models with the continuous quadratic age effect and the best random effects structure, the most parsimonious model contained effects of population density, WNAO, reproductive status, and lamb status; also age interacted with reproductive status, and lamb status interacted with population density (Table 1). General results were consistent across the different random effects structures (appendix S4). See online appendix S2 for details of the most parsimonious model.

Reproductive individuals had lower growth rates than non-reproductive individuals and the effect was greater in adults than in lambs (Figure 2). For a median female, the cost of reproduction amounted to $0.5 \mathrm{~kg}$, i.e. $2 \%$ of their body mass or $46 \%$ of their expected growth if not reproductive. High population density reduced growth rates for all ages, but especially for lambs (Figure 3). Higher WNAO increased growth rates (Figure 4).

Persistent heterogeneity among individuals (CV 0.027, 0.001 SE) was larger than process error (CV 0.018, $0.0008 \mathrm{SE}$ ) and heterogeneity among years (CV 0.01, 0.002 SE) and birth cohorts (CV 0.005, $0.002 \mathrm{SE}$ ). Individual deviations from the average growth trajectory 
were positively correlated with maximum mass $\left(0.23, \mathrm{p}<10^{-15}\right)$, longevity $\left(0.13, \mathrm{p}<10^{-6}\right)$, lifetime reproduction $\left(0.18, \mathrm{p}<10^{-9}\right)$, and reproduction in the first year of life $(\mathrm{p}<0.01)$. In our comparison of a LMM with our most parsimonious state-space model of sheep growth, the LMM was able to use 2530 observations amounting to 1799 observed growth increments - far fewer than the 4038 mass observations used in the state-space model. Despite sample size differences, the LMM and state-space model had quite similar parameter estimates (appendix S2). The LMM had a lower estimated intercept, a lower (more negative) effect of age, a stronger effect of WNAO, and a higher slope on previous size. Only the age effect had confidence intervals that did not overlap the estimate from the statespace model (appendix S2).

\section{Speed of growmod}

The most parsimonious model, which was highly parameterized relative to other formulas, took 20 seconds to fit using our method. The corresponding LMM, with the same fixed and random effects as in eqn 1, could be fit using the lmer function in 0.2 seconds. Using 7 computer cores in parallel, fitting 4466 models with different versions of the fixed effects and the all of the random effects considered here took 6.9 hours, i.e., 6 seconds per model on average.

\section{Discussion}

In this study, using our method applied to simulated data, we showed that statespace models are less biased than LMMs for estimating AR(1) growth patterns. In comparison with other modeling approaches, our method is more flexible than size-at-age models and much faster than MCMC sampling methods. We applied both LMMs and statespace models to a well-studied population and showed that, while both models gave similar results, the LMM's accuracy was due to two sources of bias negating each other. Our simulation study demonstrates that this coincidence should not occur commonly, and thus 
LMM's should not be viewed as equivalent in accuracy to our state-space approach. For the first time, we have shown that female Soay sheep incur an immediate cost of reproduction on annual growth of $2 \%$ of their body mass or $46 \%$ of their expected growth. Furthermore, we showed that growth is reduced at high population densities and low winter NAO values.

\section{Comparison with other methodologies}

LMMs can be used to model an AR(1) growth process, but our simulation study showed that imperfect recapture rates and observation error cause severe biases for LMMs. Estimates of individual heterogeneity were increasingly biased downward for lower recapture rates and increasingly biased upward with larger observation error. Observation error increased the age-specific intercept and reduced the age-specific slope on previous mass; this agrees with the statistical principle that adding noise to data attenuates the estimated slope. Imperfect recapture caused biases in the opposite direction from observation error and in some studies, these biases may negate each other. However, this coincidental negation is more likely to be the exception than the rule and under most circumstances, the resulting estimates will be severely biased, as we have shown in the simulation study. When there is substantial bias in estimated LMM coefficients, it is difficult to intuit the implications for predictive models that use those biased coefficients. That's because a positive bias in the intercept combined with a negative bias in the slope on previous mass may have negligible consequences for most masses in the observed range.

Our modeling approach allowed us to account for the effects of persistent and transient heterogeneity that may have lasting effects throughout life. Size-at-age models can be adapted to account for the environment (English et al. 2011; Huchard et al. 2014) and transient individual heterogeneity (Webber and Thorson 2015). However, estimated effects of previous environments are not persistent in size-at-age models and could be confounded with other effects such as persistent or transient individual heterogeneity. Our AR(1) 
approach includes the carryover effects of past environments on growth trajectories. For example, lambs that experience a high population density in their first year of life will have a lower size the following year and this will carry over with an effect that diminishes based on the autoregressive part of the model ( $M \eta$ in eqn 1). This lasting effect of past environments can have important repercussions at the population level (Ozgul et al. 2009). It may also affect population dynamics because survival and reproduction are associated with mass.

Using TMB allowed us to achieve the flexibility and accuracy of state-space models at a speed that is comparable to LMMs. If we assume this model follows the published pattern (Pedersen et al. 2011; Bolker et al. 2013; Kristensen et al. 2016), then using MCMC sampling methods would require approximately 20 to 150 days to select the fixed effects given the continuous age effect; other age effects and random effects structures would require additional time. Doing model selection either by an exhaustive search, as we have done here, or by stepwise selection is important for finding the set of variables that give the best predictive ability or the highest explanatory power (Murtaugh 2009). Access to fast and flexible methods gives biologists greater ability to test more forms of their model and find the best one, whereas slower methods would hinder the selection process.

\section{Comparison with previous studies of Soay sheep}

The general results from our model are similar to previous studies of the same population of Soay sheep, but our results on reproduction differed. We found that the cost to lambs was smaller than the cost to adults whereas Ozgul et al. (2009) only detected a significant cost in lambs. The observed cost to lambs may be small if those that cannot pay the growth cost of reproduction die over the winter, especially since there is a survival cost of reproduction in this population which is strongest for individuals of ages one year or over seven years (Tavecchia et al. 2005). The survival cost means that we observe a truncated 
portion of the population. Estimates of the growth costs of reproduction were similar in both the state-space model and the LMM of the same form. Ozgul et al. (2009) found a trend for a positive effect of reproduction on growth of adults and senescent individuals. Because our LMM and state-space model of sheep growth gave similar results to each other, we conclude that the differences between our findings and those of Ozgul et al. (2009) are probably due to the eight years of additional data in this analysis.

Post-hoc analyses showed that the individual growth deviations are positively correlated with other fitness measures (longevity, early reproduction, and lifetime reproduction). This agrees with previous findings that sheep that grow larger tend to live longer (Nussey et al. 2011). These correlations suggest that the growth deviations are capturing aspects of individual quality (Wilson and Nussey 2009), possibly relating to the positive genetic correlation between mass and parasite resistance in this population (Coltman et al. 2001). However, caution should be used in post-hoc interpretations because individual deviations are biased towards zero (Postma 2006; Hadfield et al. 2010); a thorough assessment should estimate correlations within the estimation procedure.

Costs of reproduction are foundational to the study of life-history evolution. Thus the cost of reproduction on growth that we detected is likely to have evolutionary consequences for the life history of sheep because survival and reproduction depend on mass (Clutton-Brock et al. 1996; Milner et al. 1999). Current reproduction that reduces a female's future mass will also reduce her future survival and reproduction. Consequences of the growth cost may be subtler than the survival cost demonstrated by Tavecchia et al. (2005). Previous predictions of the optimal reproductive allocation in Soay sheep included reduced survival rates of reproductive females but did not account for the growth costs observed here (Clutton-Brock et al. 1996; Marrow et al. 1996; Wilson et al. 2009; Childs et al. 2011). Positive selection on offspring mass and number may be counter-balanced by 
selection on adult females to have a high mass leading into the winter when mortality risk is high. Future work on optimal allocation in Soay sheep should account for these growth costs.

\section{Conclusion}

Our approach of modeling growth in a state-space framework is flexible enough to include environmental effects and avoids biases caused by observation error and missing repeated measures - common problems in studies of natural populations. We have shown that biases from these two sources act in opposite directions such that, in certain combinations, they can negate each other almost completely, as in the Soay sheep case study. However, we recommend against using LMMs when more than $25 \%$ of repeated measures are missing, as previously recommended by Hamel et al. (2012). We have introduced a new tool (growmod) for avoiding these biases while estimating individual growth trajectories much faster than was previously possible. Better estimates will give us more power to make predictions at higher levels of organization because patterns of individual growth have consequences for eco-evolutionary dynamics.

\section{Acknowledgements}

Many thanks to Dan Nussey for helpful comments on earlier drafts of the manuscript; to Erik Postma and Ben Bolker for helpful discussion about BLUPs; to Jarrod Hadfield for advice on back-transforming variables from the log scale; to Stefanie Muff and Michael Morrissey for discussion about observation error; to Hans Skaug and Kasper Kristensen for guidance with Tмв. Thanks to the National Trust for Scotland, Scottish Natural Heritage, the Ministry of Defense, QinetiQ, Amey, and ESS staff on St. Kilda and Benbecula. Thanks to Jill Pilkington, field assistants, and many volunteers who collected the long-term sheep data; this work would not have been possible without their dedicated efforts. Thanks to Tim 
Clutton-Brock for keeping the St. Kilda Soay Sheep Project going for 30 years. Thanks to the Natural Environment Research Council for funding the long-term data collection. This

research was supported by a European Research Council Starting Grant \#337785 to AO, and Swiss National Science Foundation Grants 31003A_146445 to AO and IZ320Z0_161670 to MEB.

\section{References}

Arnold, S. J. 1981. Behavioral Variation in Natural Populations. II. The Inheritance of a Feeding Response in Crosses Between Geographic Races of the Garter Snake, Thamnophis elegans. Evolution 35:510-515.

Bates, D., M. Mächler, B. M. Bolker, and S. Walker. 2015a. Fitting Linear Mixed-Effects Models Using lme4. Journal of Statistical Software 67:1-48.

Bates, D., R. Kliegl, S. Vasishth, and H. Baayen. 2015b. Parsimonious Mixed Models. arXiv:1506.04967.

Bengtsson, T., and J. E. Cavanaugh. 2006. An improved Akaike information criterion for state-space model selection. Computational statistics \& data analysis 50:2635-2654.

Bertalanffy, Von, L. 1957. Quantitative laws in metabolism and growth. Quarterly Review of Biology 32: 217-231.

Bolker, B. M. 2015. Linear and Generalized Linear Mixed Models. in G. A. Fox, S. NegreteYankelevich, and V. J. Sosa, eds. Ecological Statistics. Oxford University Press, Oxford, UK.

Bolker, B. M., B. Gardner, M. Maunder, C. W. Berg, M. Brooks, L. Comita, E. Crone, et al. 2013. Strategies for fitting nonlinear ecological models in R, AD Model Builder, and BUGS. Methods in Ecology and Evolution 4:501-512.

Bonnet, T., and E. Postma. 2016. Successful by Chance? The Power of Mixed Models and 
Neutral Simulations for the Detection of Individual Fixed Heterogeneity in Fitness Components. The American Naturalist 187:60-74.

Brienen, R., P. A. Zuidema, and H. During. 2006. Autocorrelated growth of tropical forest trees: unraveling patterns and quantifying consequences. Forest Ecology and Management 237:179-190.

Brooks, M. E., M. W. Mccoy, and B. M. Bolker. 2013. A Method for Detecting Positive Growth Autocorrelation without Marking Individuals. PLoS ONE 8:e76389.

Brooks, M. E., C. Clements, J. M. Pemberton, and A. Ozgul. 2017. Data from: Estimation of individual growth trajectories when repeated measures are missing. Dryad Digital Repository. http://dx.doi.org/10.5061/dryad.r6j80

Cam, E., O. Gimenez, R. Alpizar-Jara, L. M. Aubry, M. Authier, E. G. Cooch, D. N. Koons, et al. 2012. Looking for a needle in a haystack: inference about individual fitness components in a heterogeneous population. Oikos 122:739-753.

Carroll, R. J., D. Ruppert, L. A. Stefanski, and C. M. Crainiceanu. 2006. Measurement Error in Nonlinear Models. CRC Press.

Cavanaugh, J. E., and R. H. Shumway. 1997. A bootstrap variant of AIC for state-space model selection. Statistica Sinica 7:473-496.

Cheng, Y. W., and A. Kuk. 2002. Determination of the unknown age at first capture of western rock lobsters (Panulirus cygnus) by random effects model. Biometrics $58: 459-462$.

Chevin, L.-M. 2015. Evolution of adult size depends on genetic variance in growth trajectories: a comment on analyses of evolutionary dynamics using integral projection models. Methods in Ecology and Evolution 6:981-986.

Childs, D. Z., B. C. Sheldon, and M. Rees. 2016. The evolution of labile traits in sex- and agestructured populations. The Journal of Animal Ecology 85:329-342. 
Childs, D. Z., T. N. Coulson, J. M. Pemberton, T. H. Clutton-Brock, and M. Rees. 2011.

Predicting trait values and measuring selection in complex life histories: reproductive allocation decisions in Soay sheep. Ecology Letters 14:985-992.

Clark, J. S., M. Wolosin, M. Dietze, I. Ibáñez, S. LaDeau, M. Welsh, and B. Kloeppel. 2007. Tree growth inference and prediction from diameter censuses and ring widths. Ecological Applications 17:1942-1953.

Clutton-Brock, T. H., and J. M. Pemberton. 2004. Soay Sheep. Cambridge University Press.

Clutton-Brock, T. H., I. R. Stevenson, and P. Marrow. 1996. Population fluctuations, reproductive costs and life-history tactics in female Soay sheep. Journal of Animal Ecology 65:675-689.

Coleman, K., and D. Wilson. 1998. Shyness and boldness in pumpkinseed sunfish: individual differences are context-specific. Animal Behaviour 56:927-936.

Coltman, D. W., J. Pilkington, L. E. Kruuk, K. Wilson, and J. M. Pemberton. 2001. Positive genetic correlation between parasite resistance and body size in a free-living ungulate population. Evolution 55:2116-2125.

de Valpine, P. 2009. Shared challenges and common ground for Bayesian and classical analysis of hierarchical statistical models. Ecological Applications 19:584-588.

de Valpine, P., and A. Hastings. 2002. Fitting population models incorporating process noise and observation error. Ecological Monographs 72:57-76.

de Valpine, P., K. Scranton, J. Knape, K. Ram, and N. J. Mills. 2014. The importance of individual developmental variation in stage-structured population models. Ecology Letters 17:1026-1038.

Dennis, B., J. M. Ponciano, and M. L. Taper. 2010. Replicated sampling increases efficiency in monitoring biological populations. Ecology 91:610-620.

Dennis, B., J. M. Ponciano, S. R. Lele, M. L. Taper, and D. F. Staples. 2006. Estimating density 
dependence, process noise, and observation error. Ecological Monographs 76:323341.

Easterling, M. R., S. P. Ellner, and P. M. Dixon. 2000. Size-specific sensitivity: applying a new structured population model. Ecology 81:694-708.

Emmerson, M. C., and D. Raffaelli. 2004. Predator-prey body size, interaction strength and the stability of a real food web. Journal of Animal Ecology 73:399-409.

English, S., A. W. Bateman, and T. H. Clutton-Brock. 2011. Lifetime growth in wild meerkats: incorporating life history and environmental factors into a standard growth model. Oecologia 169:143-153.

Ford, E. J. 1933. An account of the herring investigations conducted at Plymouth during the years from 1924 to 1933. Journal of the Marine Biological Association of the United Kingdom 19:305-384.

Hadfield, J. D., A. J. Wilson, D. Garant, B. C. Sheldon, and L. E. B. Kruuk. 2010. The Misuse of BLUP in Ecology and Evolution. The American Naturalist 175:116-125.

Hadfield, J. D., E. A. Heap, F. Bayer, E. A. Mittell, and N. M. A. Crouch. 2013. Disentangling Genetic and Prenatal Sources of Familial Resemblance Across Ontogeny in a Wild Passerine. Evolution 67:2701-2713.

Hamel, S., J. M. Gaillard, N. G. Yoccoz, S. Albon, S. D. Cote, J. M. Craine, M. Festa-Bianchet, et al. 2016. Cohort variation in individual body mass dissipates with age in large herbivores. Ecological Monographs 86: 517-543.

Hamel, S., N. G. Yoccoz, and J.-M. Gaillard. 2012. Statistical evaluation of parameters estimating autocorrelation and individual heterogeneity in longitudinal studies. Methods in Ecology and Evolution 3:731-742.

Hedrick, P. W., D. W. Coltman, M. Festa-Bianchet, and F. Pelletier. 2014. Not surprisingly, no inheritance of a trait results in no evolution. Proceedings of the National Academy of 
Sciences 111:E4810-E4810.

Horton, N. J., and K. P. Kleinman. 2007. Much Ado About Nothing. The American Statistician 61:79-90.

Huchard, E., A. Charmantier, S. English, A. W. Bateman, J. F. Nielsen, and T. Clutton-Brock. 2014. Additive genetic variance and developmental plasticity in growth trajectories in a wild cooperative mammal. Journal of Evolutionary Biology 27:1893-1904.

Kendall, B. E., G. A. Fox, M. Fujiwara, and T. M. Nogeire. 2011. Demographic heterogeneity, cohort selection, and population growth. Ecology 92:1985-1993.

Kitagawa, G. 1987. Non-Gaussian state-space modeling of nonstationary time series. Journal of the American Statistical Association 82:1032-1041.

Kristensen, K., A. Nielsen, C. W. Berg, H. J. Skaug, and B. M. Bell. 2016. TMB: Automatic Differentiation and Laplace Approximation. Journal of Statistical Software 70:1-21.

Lindström, J., and H. Kokko. 2002. Cohort effects and population dynamics. Ecology Letters $5: 338-344$.

Lorenzen, K. 2016. Toward a new paradigm for growth modeling in fisheries stock assessments: Embracing plasticity and its consequences. Fisheries Research 180:422.

Marrow, P., J. M. McNamara, A. I. Houston, I. R. Stevenson, and T. H. Clutton-Brock. 1996. State-dependent life history evolution in Soay sheep: dynamic modelling of reproductive scheduling. Philosophical transactions of the Royal Society of London. Series B, Biological sciences 351:17-32.

Milner, J. M., D. A. Elston, and S. D. Albon. 1999. Estimating the contributions of population density and climatic fluctuations to interannual variation in survival of Soay sheep. Journal of Animal Ecology 68:1235-1247.

Morgan, T. J., T. Garland, and P. A. Carter. 2003. Ontogenies in mice selected for high 
voluntary wheel-running activity. I. Mean ontogenies. Evolution 57:646-657.

Murtaugh, P. A. 2009. Performance of several variable-selection methods applied to real ecological data. Ecology Letters 12:1061-1068.

Nakagawa, S., and R. P. Freckleton. 2008. Missing inaction: the dangers of ignoring missing data. Trends in Ecology \& Evolution 23:592-596.

Nussey, D. H., T. Coulson, D. Delorme, T. H. Clutton-Brock, J. M. Pemberton, M. FestaBianchet, and J.-M. Gaillard. 2011. Patterns of body mass senescence and selective disappearance differ among three species of free-living ungulates. Ecology 92:19361947.

O'Connor, C. M., D. R. Norris, G. T. Crossin, and S. J. Cooke. 2014. Biological carryover effects: linking common concepts and mechanisms in ecology and evolution. Ecosphere 5:111.

Ozgul, A., A. W. Bateman, S. English, T. Coulson, and T. H. Clutton-Brock. 2014. Linking body mass and group dynamics in an obligate cooperative breeder. Journal of Animal Ecology 83:1357-1366.

Ozgul, A., S. Tuljapurkar, T. G. Benton, J. M. Pemberton, T. H. Clutton-Brock, and T. Coulson. 2009. The Dynamics of Phenotypic Change and the Shrinking Sheep of St. Kilda. Science 325:464-467.

Pedersen, M. W., C. W. Berg, U. H. Thygesen, A. Nielsen, and H. Madsen. 2011. Estimation methods for nonlinear state-space models in ecology. Ecological Modelling 222:13941400.

Pfister, C. A., and M. Wang. 2005. Beyond size: matrix projection models for populations where size is an incomplete descriptor. Ecology 86:2673-2683.

Pfister, C., and F. Stevens. 2002. The genesis of size variability in plants and animals. Ecology 83:59-72. 
Plard, F., J.-M. Gaillard, T. Coulson, D. Delorme, C. Warnant, J. Michallet, S. Tuljapurkar, et al. 2015. Quantifying the influence of measured and unmeasured individual differences on demography. Journal of Animal Ecology 84:1434-1445.

Pletcher, S. D., and C. J. Geyer. 1999. The genetic analysis of age-dependent traits: modeling the character process. Genetics 153:825-835.

Postma, E. 2006. Implications of the difference between true and predicted breeding values for the study of natural selection and micro-evolution. Journal of Evolutionary Biology 19:309-320.

R Core Development Team. 2016. R: A Language and Environment for Statistical Computing. R Foundation for Statistical Computing, Vienna, Austria.

Sainsbury, K. J. 1980. Effect of individual variability on the von Bertalanffy growth equation. Canadian Journal of Fisheries and Aquatic Sciences 37:241-247.

Salguero-Gómez, R., and B. B. Casper. 2010. Keeping plant shrinkage in the demographic loop. Journal of Ecology 98:312-323.

Shelton, A. O., and M. Mangel. 2012. Estimating von Bertalanffy parameters with individual and environmental variations in growth. Journal of Biological Dynamics 6:3-30.

Shelton, A. O., W. H. Satterthwaite, M. P. Beakes, S. B. Munch, S. M. Sogard, and M. Mangel. 2013. Separating Intrinsic and Environmental Contributions to Growth and Their Population Consequences. The American Naturalist 181:799-814.

Sigourney, D. B., S. B. Munch, and B. H. Letcher. 2012. Combining a Bayesian nonparametric method with a hierarchical framework to estimate individual and temporal variation in growth. Ecological Modelling 247:125-134.

Sih, A., A. Bell, and J. Johnson. 2004. Behavioral syndromes: an ecological and evolutionary overview. Trends in Ecology \& Evolution 19:372-378.

Simmonds, E. G. and Coulson, T. 2014. Analysis of phenotypic change in relation to climatic 
drivers in a population of Soay sheep Ovis aries, 124:543-552.

Sofaer, H. R., P. L. Chapman, T. S. Sillett, and C. K. Ghalambor. 2013. Advantages of nonlinear mixed models for fitting avian growth curves. Journal of Avian Biology 44:469-478.

Stinchcombe, J. R., and M. Kirkpatrick. 2012. Genetics and evolution of function-valued traits: understanding environmentally responsive phenotypes. Trends in Ecology \& Evolution 27:637-647.

Szalai, E. B., G. W. Fleischer, and J. R. Bence. 2003. Modeling time-varying growth using a generalized von Bertalanffy model with application to bloater (Coregonus hoyi) growth dynamics in Lake Michigan. Canadian Journal of Fisheries and Aquatic Sciences 60:55-66.

Tavecchia, G., P. Besbeas, T. Coulson, B. J. T. Morgan, and T. H. Clutton-Brock. 2009. Estimating Population Size and Hidden Demographic Parameters with State-Space Modeling. The American Naturalist 173:722-733.

Tavecchia, G., T. Coulson, and B. Morgan. 2005. Predictors of reproductive cost in female Soay sheep. Journal of Animal Ecology 74:201-213.

Thorson, J. T., C. C. Monnahan, and J. M. Cope. 2015a. The potential impact of time-variation in vital rates on fisheries management targets for marine fishes. Fisheries Research 169:8-17.

Thorson, J. T., J. N. Ianelli, and S. B. Munch. 2015b. Spatial delay-difference models for estimating spatiotemporal variation in juvenile production and population abundance. Canadian Journal of Fisheries and Aquatic Sciences.

Tuljapurkar, S., U. K. Steiner, and S. H. Orzack. 2009. Dynamic heterogeneity in life histories. Ecology Letters 12:93-106.

Vaida, F., and S. Blanchard. 2005. Conditional Akaike information for mixed-effects models. Biometrika 92:351-370. 
Vincenzi, S., M. Mangel, A. J. Crivelli, S. Munch, and H. J. Skaug. 2014. Determining Individual Variation in Growth and Its Implication for Life-History and Population Processes Using the Empirical Bayes Method. PLoS Computational Biology 10:e1003828.

Vindenes, Y., and Ø. Langangen. 2015. Individual heterogeneity in life histories and ecoevolutionary dynamics. Ecology Letters 18:417-432.

Walford, L. A. 1946. A new graphic method of describing the growth of animals. The Biological bulletin 90:141-147.

Wearing, H. J., P. Rohani, T. C. Cameron, and S. M. Sait. 2004. The dynamical consequences of developmental variability and demographic stochasticity for host-parasitoid interactions. The American Naturalist 164:543-558.

Webber, D. N., and J. T. Thorson. 2015. Variation in growth among individuals and over time: A case study and simulation experiment involving tagged Antarctic toothfish. Fisheries Research 180:67-76.

Weiner, J. 1985. Size hierarchies in experimental populations of annual plants. Ecology $66: 743-752$.

Wikelski, M., and C. Thom. 2000. Marine iguanas shrink to survive El Niño. Nature 403:3738.

Wilson, A. J., and D. H. Nussey. 2009. What is individual quality? An evolutionary perspective. Trends in Ecology \& Evolution 25:207-214.

Wilson, A. J., D. Réale, M. N. Clements, M. M. Morrissey, E. Postma, C. A. Walling, L. E. B. Kruuk, et al. 2010. An ecologist's guide to the animal model. Journal of Animal Ecology 79:13-26.

Wilson, A. J., J. M. Pemberton, J. G. Pilkington, T. H. Clutton-Brock, and L. E. B. Kruuk. 2009. Trading offspring size for number in a variable environment: selection on reproductive investment in female Soay sheep. Journal of Animal Ecology 78:354- 
364.

Zuidema, P. A., R. J. W. Brienen, H. J. During, and B. Güneralp. 2009. Do Persistently Fast-

Growing Juveniles Contribute Disproportionately to Population Growth? A New

Analysis Tool for Matrix Models and Its Application to Rainforest Trees. The American

Naturalist 174:709-719.

Figure legends

Figure 1. LMMs are biased by imperfect recapture rates (left column) or observation error (right column) in opposite directions. Each row of panels is a coefficient in an AR(1) growth model. Points are the estimated coefficients from 100 replicates. Colored lines are cubic splines fit to the estimates of each model. Black lines are the true value of the parameter used to simulate data.

Figure 2. Reproduction reduces growth. Lines represent predictions of the most parsimonious model for lambs (dashed) and three-year-old ewes (solid), three being the median age. Line lengths span 95\% of observed masses for these classes of individuals. Points represent observations. Color represents whether or not the female reproduced in year $t+1$. The dotted black 1-to-1 line represents stasis.

Figure 3. Increasing population density reduces growth. Lines represent predictions of the most parsimonious model for non-reproductive lambs (dashed) and reproductive three-year-old ewes (solid). Line lengths span 95\% of observed masses for these classes of individuals. Color represents predictions (lines) and observations (points) from the years with the $2^{\text {nd }}$ lowest and the highest population densities; too few observations were available to plot data from the year with the lowest population density. The dotted black 1-to-1 line represents stasis.

Figure 4. Increasing winter NAO increases growth. Lines represent predictions of the most parsimonious model for non-reproductive lambs (dashed) and reproductive three-year-old ewes (solid). Line lengths span 95\% of observed masses for these classes of individuals. Color represents predictions (lines) and observations (points) from the years with the highest and $2^{\text {nd }}$ lowest winter NAO; too few observations were available to plot data from the year with the lowest winter NAO. The dotted black 1-to-1 line represents stasis. 

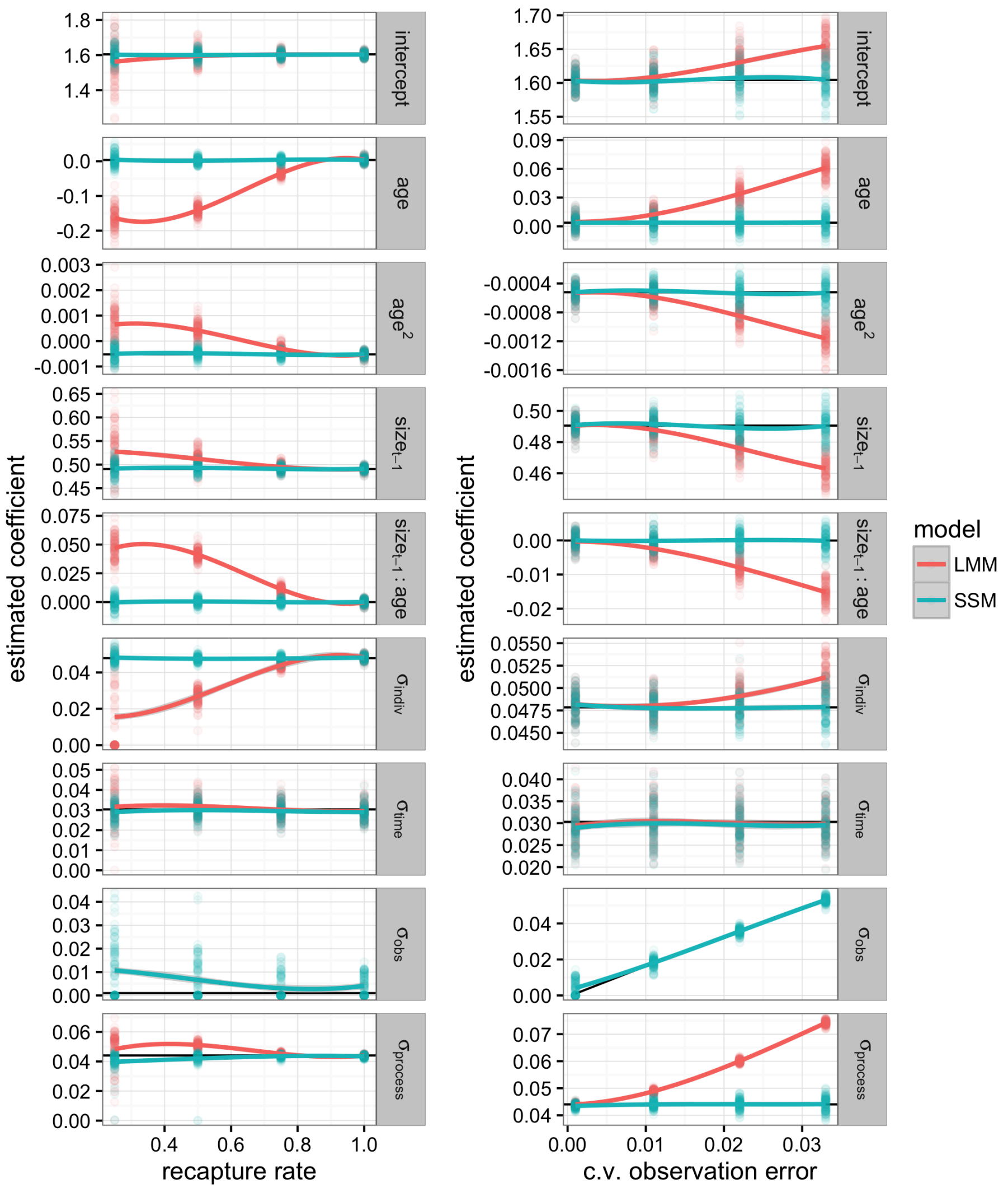


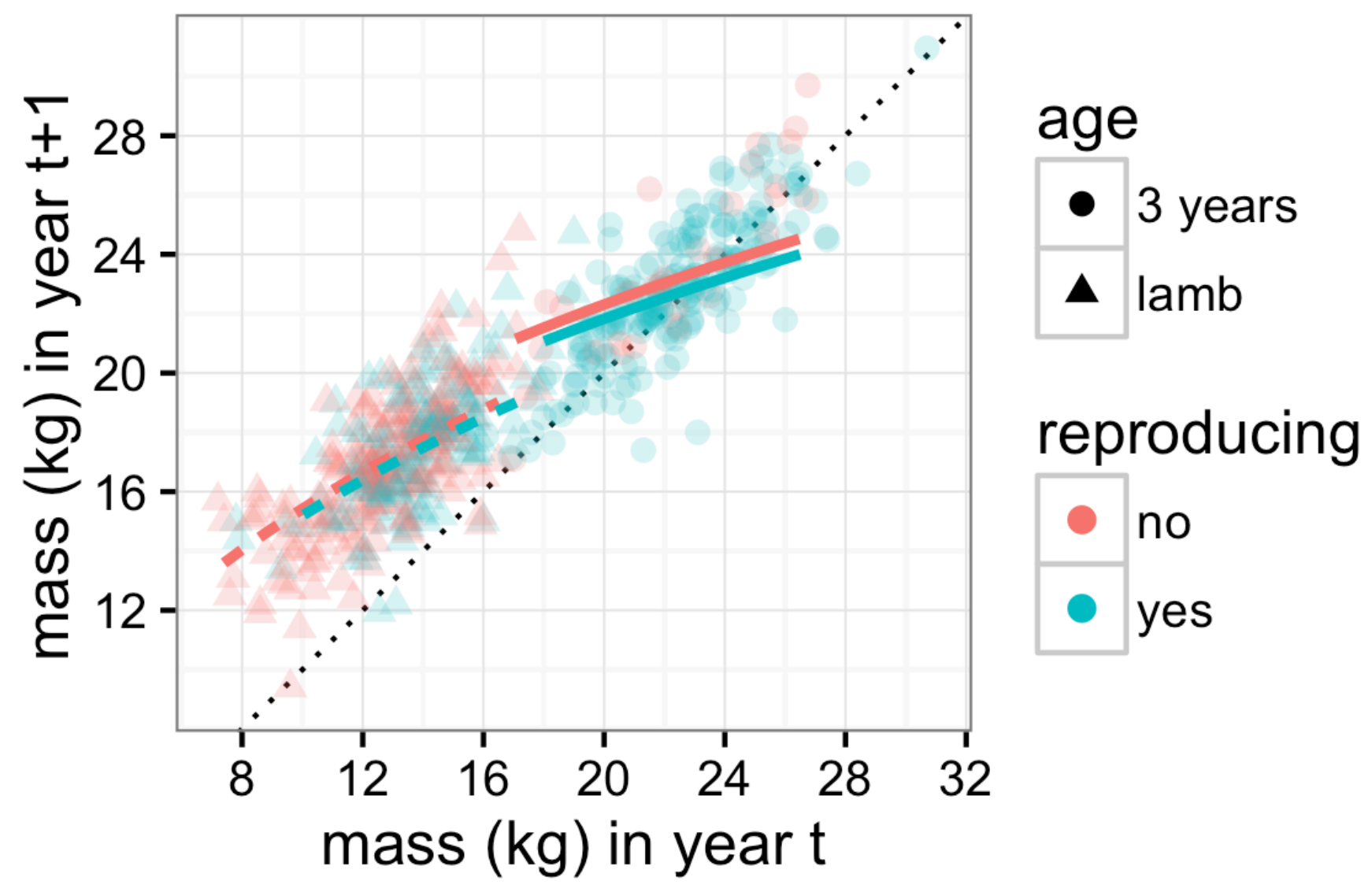




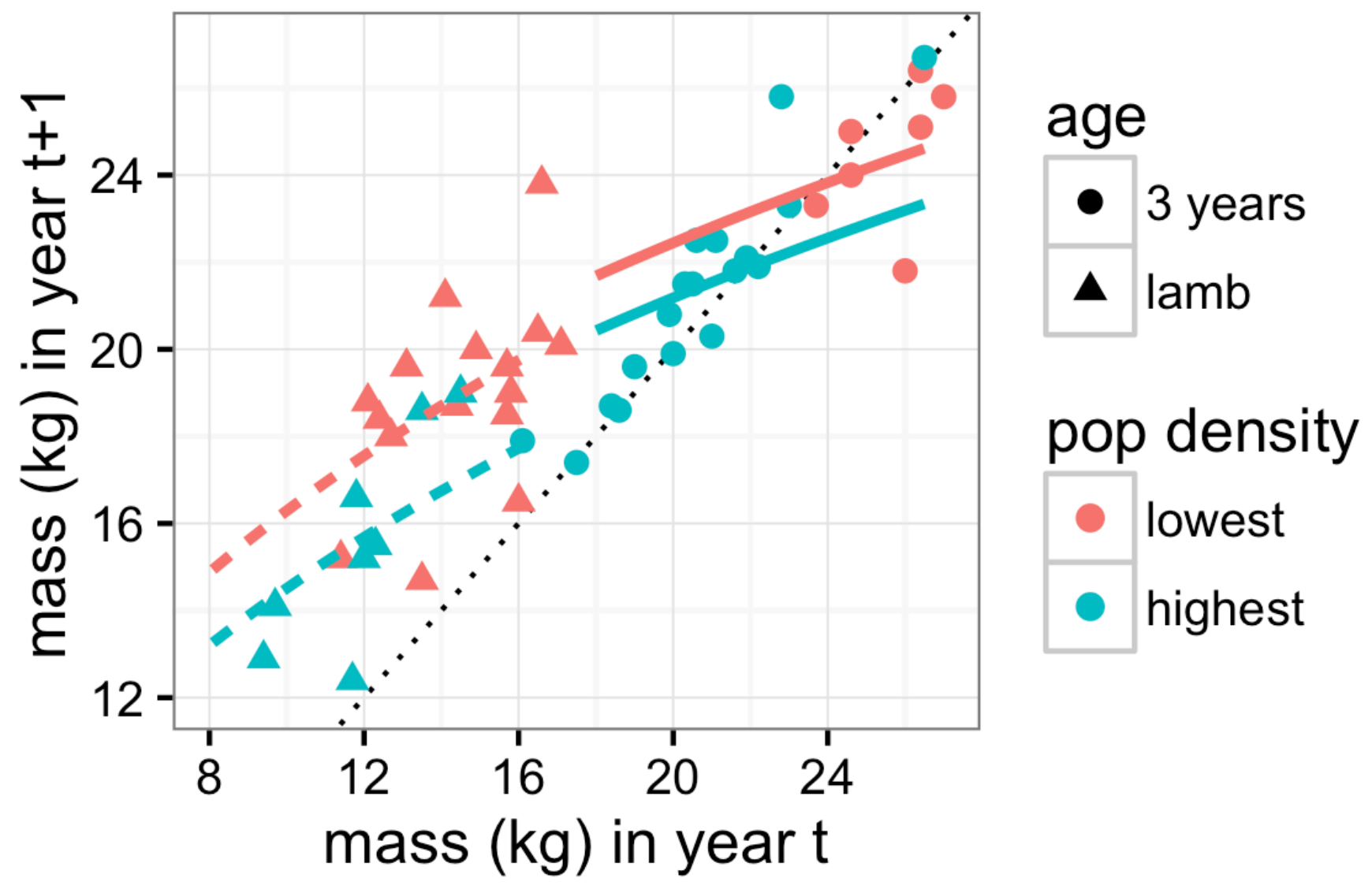




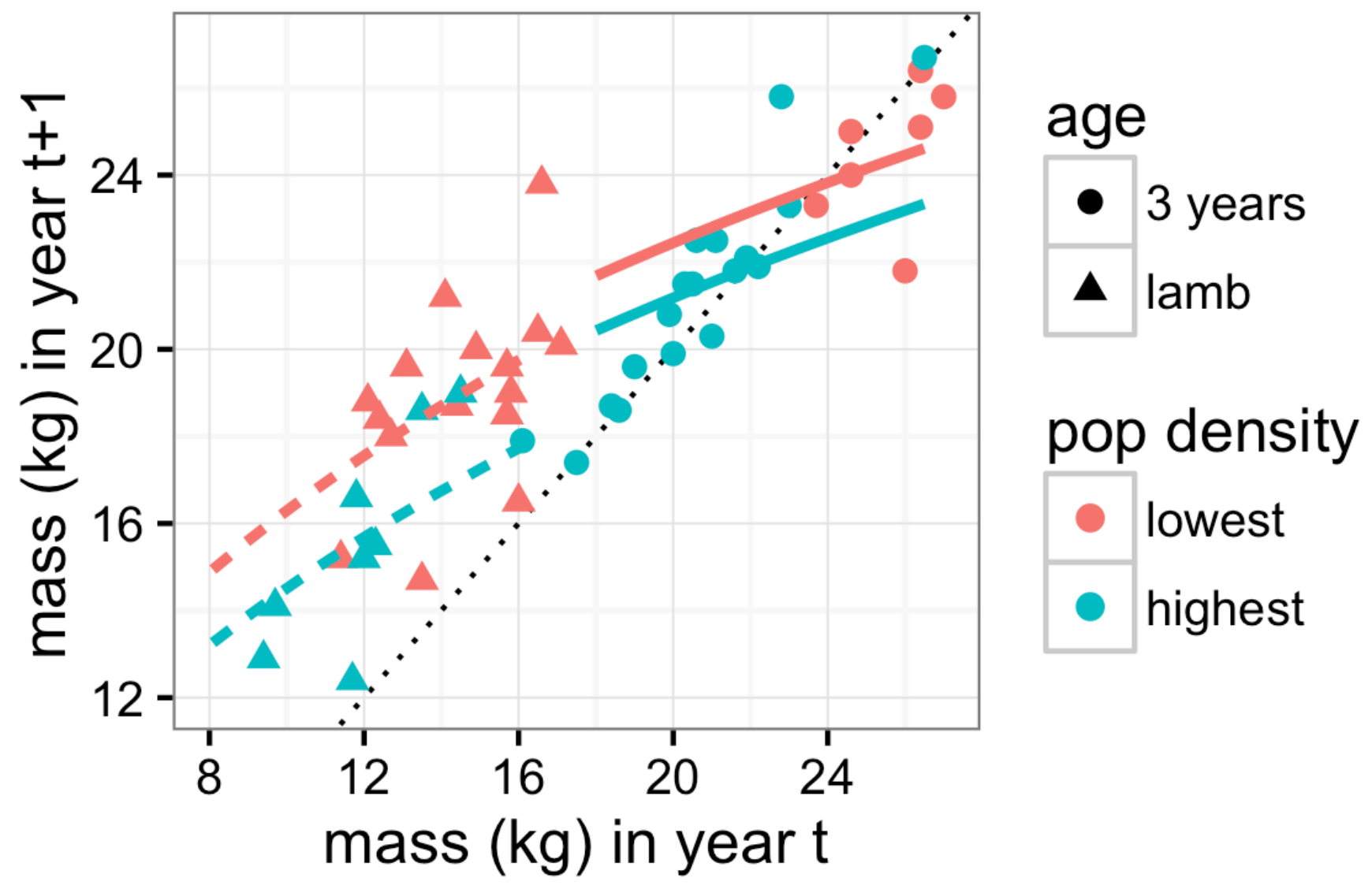




\title{
Estimation of individual growth trajectories when repeated measures are missing
}

\author{
Mollie E. Brooks ${ }^{1,2, *}$, Christopher Clements ${ }^{1}$, Josephine Pemberton ${ }^{3}$, and Arpat Ozgul ${ }^{1}$
}

${ }^{1}$ Department of Evolutionary Biology and Environmental Studies, University of Zurich, Winterthurerstrasse 190, 8057 Zurich, Switzerland ${ }^{2}$ National Institute of Aquatic Resources, Technical University of Denmark,

Charlottenlund Slot, 2920 Charlottenlund, Denmark ${ }^{3}$ Institute of Evolutionary Biology, University of

Edinburgh, Edinburgh EH9 3JT, United Kingdom

\section{Online Appendix S1 - Model with first mass covariates}

\section{Introduction}

Previous studies of growth in this population of Soay sheep separately modeled birth masses and the growth process. However, state-space models require that the initial state be modeled jointly with the process. In our Soay sheep case study, the initial state was mass in the first August of life, a few months after birth (herein referred to as "first mass"). Because maternal covariates were only available for a subset of individuals, we modeled first mass as a completely random variable in the main text. For individuals with observed first masses, their first latent mass is pulled very near to the observed mass. Only individuals with unobserved first masses have an independently estimated latent first mass. In this appendix, we describe models that were fit to the subset of the data for which we had maternal covariates available including maternal age and mass. Organizing the data is specific to this application, so it is not presented here.

\section{Defining the first mass predictors}

To choose the predictors for the first masses, we created a subset of the data that contained a row for each individual with mass observed in August at age 0 (the lamb stage) and a column for each possible predictor. This data frame only contained data for individuals at age 0 and can contain no missing values. We slected the best predictive model for first masses using the dredge function from the MuMIn package. We separated models with summer or winter NAO because they are correlated.

Then for the growth model, we needed a slightly less restricted subset of the data. It is less restriced than the data described above because it is not necessary to observe the first masses for each of these individuals, only the necessary predictors for first masses. This subset of the data is organized in datm. Unlike the data used for the simpler model, this one contains columns with the predictors of first mass with names that begin with "birth" to indicate that they were observed in the year of birth.

For conciseness, we use the following abbreviations for the coefficients. They are described in more detail in the main text.

\begin{tabular}{ll}
\hline abreviation & meaning \\
\hline a & age \\
lamb & lamb status \\
rep & reproducive status \\
pop & population density \\
nao & winter NAO \\
birth_pop & population density the year before this individual's birth \\
birth_adultmum & mother of this individual was a yearling $(0)$ or adult $(1)$ in the year of this individual's birth
\end{tabular}

\footnotetext{
*Corresponding author. email MollieEBrooks@gmail.com
} 


\begin{tabular}{ll}
\hline abreviation & meaning \\
\hline $\begin{array}{l}\text { birth_mumlogm } \\
\text { birth_twin }\end{array}$ & mass of mother in the year of this individual's birth \\
born as a twin
\end{tabular}

\section{How to use growmod}

The growmod package can be used to fit a model with predictors on initial size using the following code.

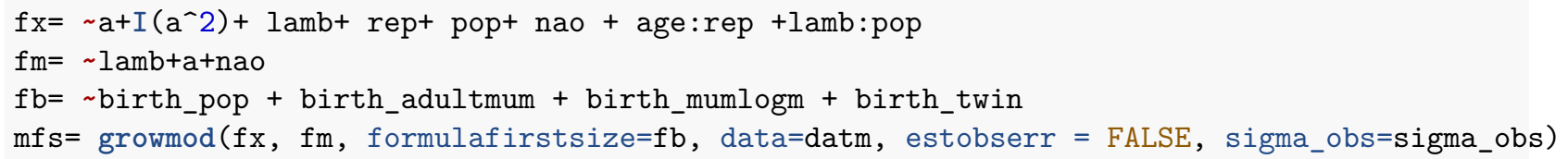

\section{Results of model selection}

View the top models fit to the subset of data for which we had the necessary predictors.

\section{First mass predicted}

\begin{tabular}{llr}
\hline formulaX & formulaM & deltaAIC \\
\hline$\sim \mathrm{a}+\mathrm{I}\left(\mathrm{a}^{\wedge} 2\right)+$ lamb+ rep+ pop+nao +lamb:pop & $\sim$ lamb+a+nao & 0.000 \\
$\sim \mathrm{a}+\mathrm{I}\left(\mathrm{a}^{\wedge} 2\right)+$ lamb+ rep+ pop+nao + a:rep +lamb:pop & $\sim$ lamb+a+nao & 0.497 \\
$\sim \mathrm{a}+\mathrm{I}\left(\mathrm{a}^{\wedge} 2\right)+$ lamb+ rep+ pop+nao + lamb:rep +lamb:pop & $\sim$ lamb+a+nao & 0.663 \\
$\sim \mathrm{a}+\mathrm{I}\left(\mathrm{a}^{\wedge} 2\right)+$ lamb+ rep+ pop+nao+lamb:nao +lamb:pop & $\sim$ lamb+a+nao & 0.740 \\
$\sim \mathrm{a}+\mathrm{I}\left(\mathrm{a}^{\wedge} 2\right)+$ lamb+ rep+ pop+nao +lamb:pop+lamb:pop:nao & $\sim$ lamb+a+nao & 1.144 \\
\hline
\end{tabular}

\section{First mass random}

\begin{tabular}{llr}
\hline formulaX & formulaM & deltaAIC \\
\hline$\sim \mathrm{a}+\mathrm{I}\left(\mathrm{a}^{\wedge} 2\right)+$ lamb+ rep+ pop+nao +lamb:pop & $\sim$ lamb+a+nao & 0.000 \\
$\sim \mathrm{a}+\mathrm{I}\left(\mathrm{a}^{\wedge} 2\right)+$ lamb+ rep+ pop+nao + a:rep +lamb:pop & $\sim$ lamb+a+nao & 0.598 \\
$\sim \mathrm{a}+\mathrm{I}\left(\mathrm{a}^{\wedge} 2\right)+$ lamb+ rep+ pop+nao+lamb:nao +lamb:pop & $\sim$ lamb+a+nao & 0.717 \\
$\sim \mathrm{a}+\mathrm{I}\left(\mathrm{a}^{\wedge} 2\right)+$ lamb+ rep+ pop+nao + lamb:rep +lamb:pop & $\sim$ lamb+a+nao & 0.847 \\
$\sim \mathrm{a}+\mathrm{I}\left(\mathrm{a}^{\wedge} 2\right)+$ lamb+ rep+ pop+nao +lamb:pop+lamb:pop:nao & $\sim$ lamb+a+nao & 1.384 \\
\hline
\end{tabular}

The top model formula is the same, but some of the other models are in a slightly different order.

Check the mean relative difference of the coefficient estimates for the top models.

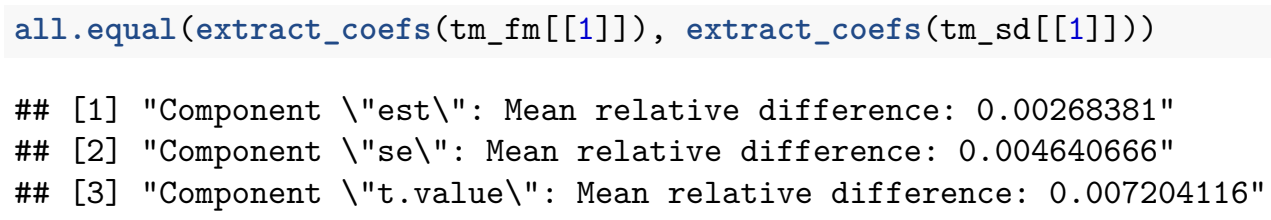

These are very small differences. So the growth estimates for the top models are essentailly equal no matter if we have predictors on mass in the first August of life or not. 


\section{Discussion}

When we restricted our data to the individuals for which first mass covariates were observed and fit models with and without these covariates on first masses, model selection chose the same model structure for either analysis of this data subset and differences in coefficient estimates were negligible $(<1 \%)$. Covariates on the first mass included population density in the previous year, maternal mass, maternal stage (yearling or not) and whether or not the individual was born as a twin. The most parsimonious model chosen for this subset of the data still showed the same general patterns. More importantly, the differences in this model compared to that chosen with the full data set are due to using a subset of the data, not due to including covariates on first masses. This indicates that the model that treats first mass as a completely random variable is adequate for the analysis of growth and its determinants. Effects on birth mass or first mass including maternal effects and inheritance can be modeled separately. 


\section{Estimation of individual growth trajectories when repeated measures are missing}

Mollie E. Brooks ${ }^{1,2, *}$, Christopher Clements ${ }^{1}$, Josephine Pemberton ${ }^{3}$, and Arpat Ozgul ${ }^{1}$

${ }^{1}$ Department of Evolutionary Biology and Environmental Studies, University of Zurich, Winterthurerstrasse 190, 8057 Zurich, Switzerland ${ }^{2}$ National Institute of Aquatic Resources, Technical University of Denmark,

Charlottenlund Slot, 2920 Charlottenlund, Denmark ${ }^{3}$ Institute of Evolutionary Biology, University of

Edinburgh, Edinburgh EH9 3JT, United Kingdom

\section{Online Appendix S2 - Examining the most parsimonious model}

In this appendix we examine aspects of the most parsimonious model. We examine the coefficient estimates and their Wald-type confidence intervals, do likelihood ratio tests on covariates of interest, and check for patterns in the random effects.

\section{The best model}

Fit as a state space model

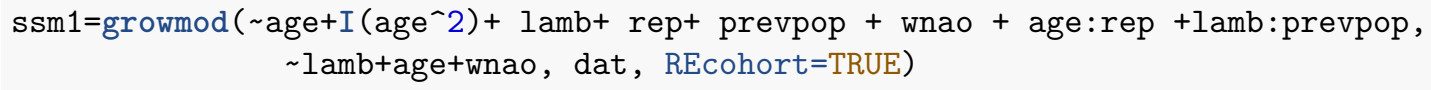

Fit as a linear mixed model

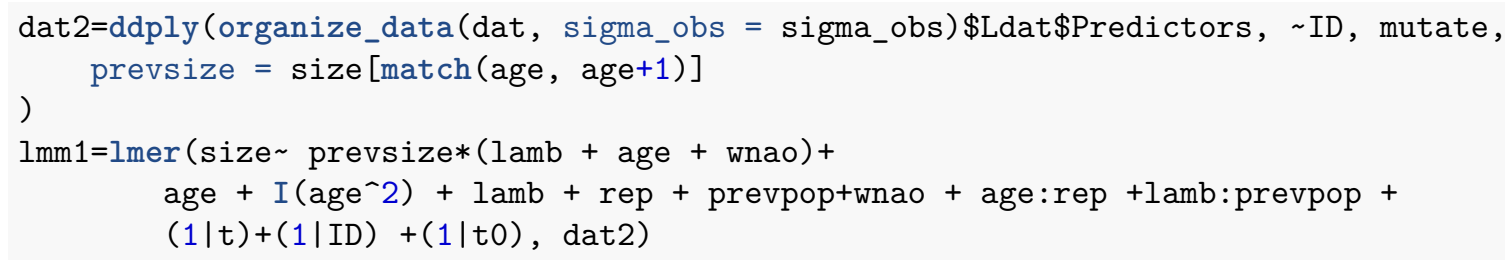

*Corresponding author. email MollieEBrooks@gmail.com 


\section{Coefficient plot}

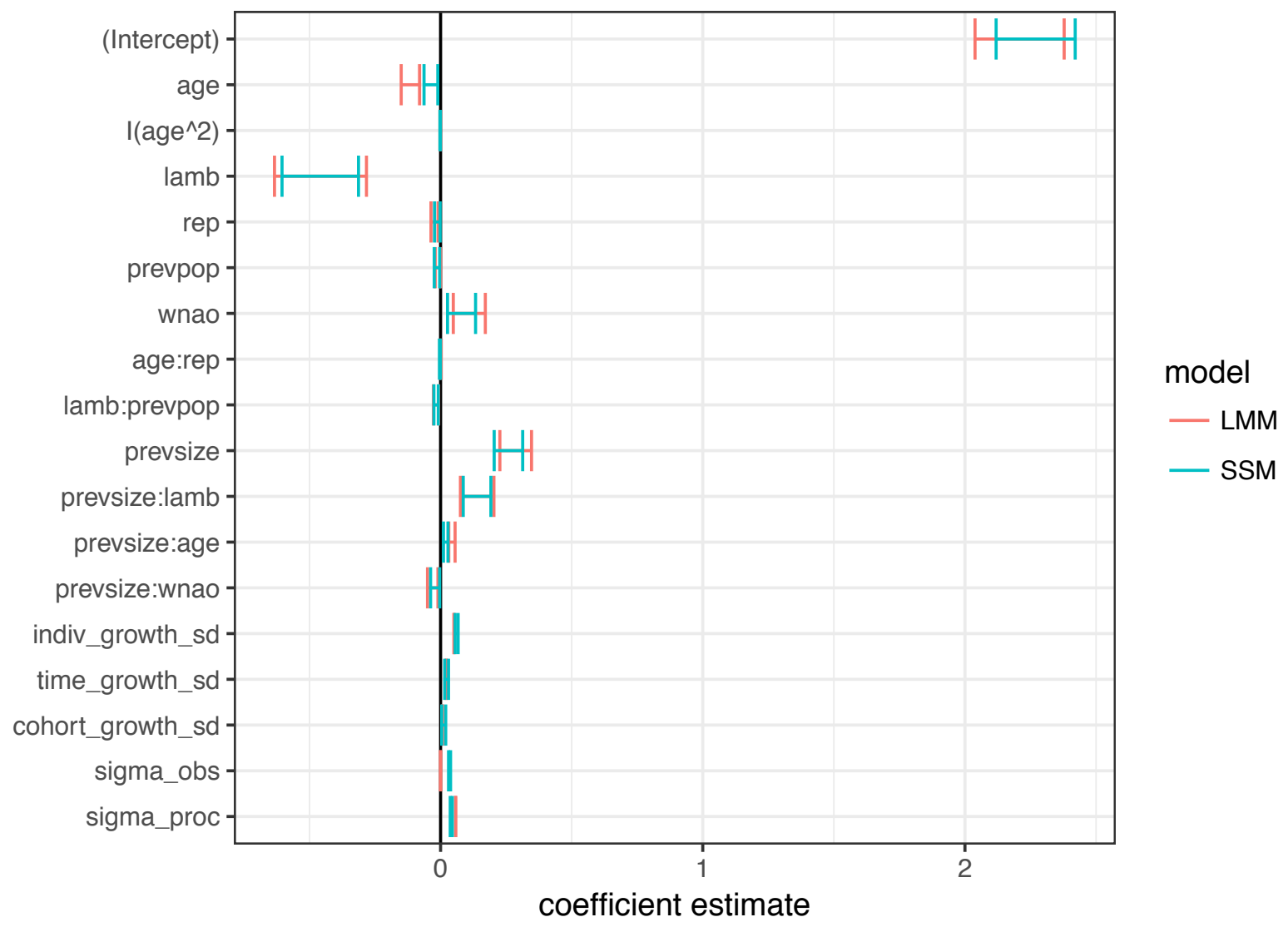

\section{Likelihood ratio tests}

For example, we can do a LRT on winter NAO using the following code.

$f x=\sim a g e+I\left(\right.$ age $\left.{ }^{-2}\right)+1 a m b+r e p+$ prevpop + age:rep +lamb:prevpop

$\mathrm{fm}=\sim$ lamb+age

wnao=growmod ( $f x, f m$, dat, REcohort=TRUE)

LRtest (ssm1, wnao)

Likelihood-ratio tests for all terms are in the following table:

\begin{tabular}{lrrr}
\hline term & statistic & df & p.value \\
\hline wnao & 11.09 & 2 & 0.0039000 \\
pop & 27.53 & 2 & 0.0000011 \\
rep & 45.93 & 2 & 0.0000000 \\
lamb & 148.47 & 3 & 0.0000000 \\
REcohort & 5.49 & 1 & 0.0191674 \\
REyear & 202.84 & 1 & 0.0000000 \\
REID & 302.29 & 1 & 0.0000000 \\
\hline
\end{tabular}




\section{Changes through time}

Here we check to see if there are any temporal patterns in the random effects. As Ozgul et al. (2009) reported that sheep have been shrinking through time, we might see some trends unless this pattern is captured by the environmental covariates of weather and populaiton density. We omit the deviations of individuals who were only observed once because they are severley shrunken toward zero.
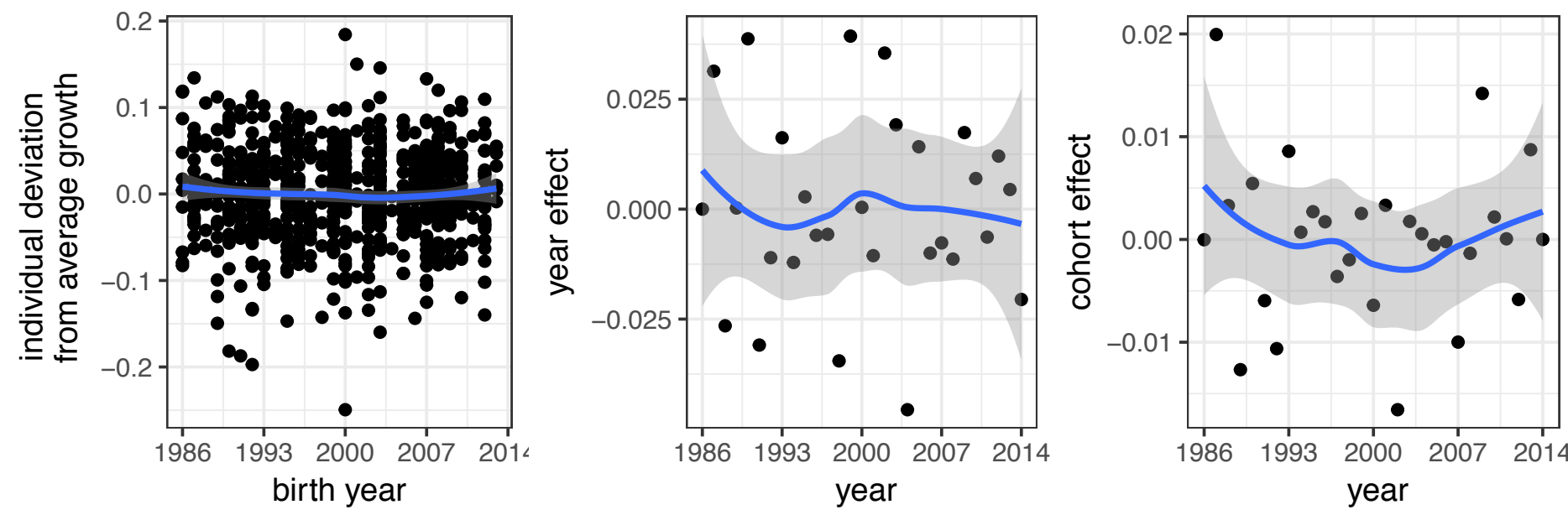

There appears to be a decreasing trend in the cohort effect for the range of years (1986 to 2006) analyzed by Ozgul et al (2009). 


\section{Estimation of individual growth trajectories when repeated measures are missing}

Mollie E. Brooks ${ }^{1,2, *}$, Christopher Clements ${ }^{1}$, Josephine Pemberton ${ }^{3}$, and Arpat Ozgul ${ }^{1}$

${ }^{1}$ Department of Evolutionary Biology and Environmental Studies, University of Zurich, Winterthurerstrasse 190, 8057 Zurich, Switzerland ${ }^{2}$ National Institute of Aquatic Resources, Technical University of Denmark,

Charlottenlund Slot, 2920 Charlottenlund, Denmark ${ }^{3}$ Institute of Evolutionary Biology, University of Edinburgh, Edinburgh EH9 3JT, United Kingdom

\section{Online Appendix S3 - Extended results of simulation study}

Here we present results of a study comparing linear mixed models and state-space models fit to simulated data sets with recapture rates (recap) of 0.05 and 0.125 and no observation error. Run vignette("sim_growmod") to see the methods. We plot the two model types in separate panels because the scales are so different. Having the models size-by-side would be better for comparisons, but this way shows more resolution.

*Corresponding author. email MollieEBrooks@gmail.com 

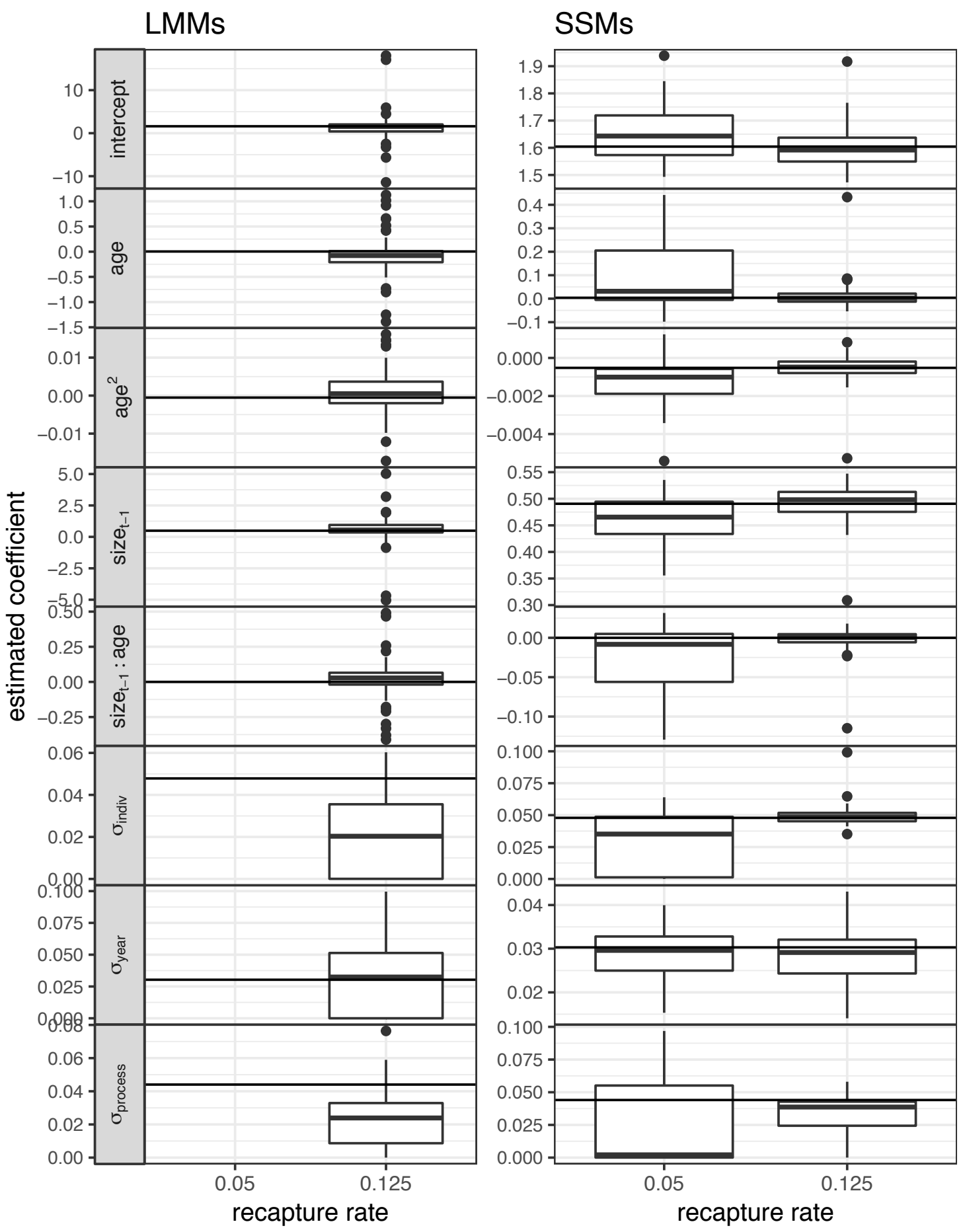

Figure S3-1 Parameter estimates. The left and right column represent linear mixed models (LMMs) and state-space models (SSMs) respectively fit to 100 simulated data sets. Data sets were simulated with recapture rates of 0.05 and 0.125 (i.e., $95 \%$ and $87.5 \%$ of repeated measures missing) which are represented by the x-axis. Each row of panels represents a different parameter of the model. Horisontal lines represent the true parameter value used to simulate growth data. Estimates are summarized by Tukey style boxplots in which boxes encompass the 25 th to the 75 th quantile with a thicker line at the median, whiskers extend from the box up to 1.5 times the inner quartile range (truncated to the location of data), and points represent outliers. 

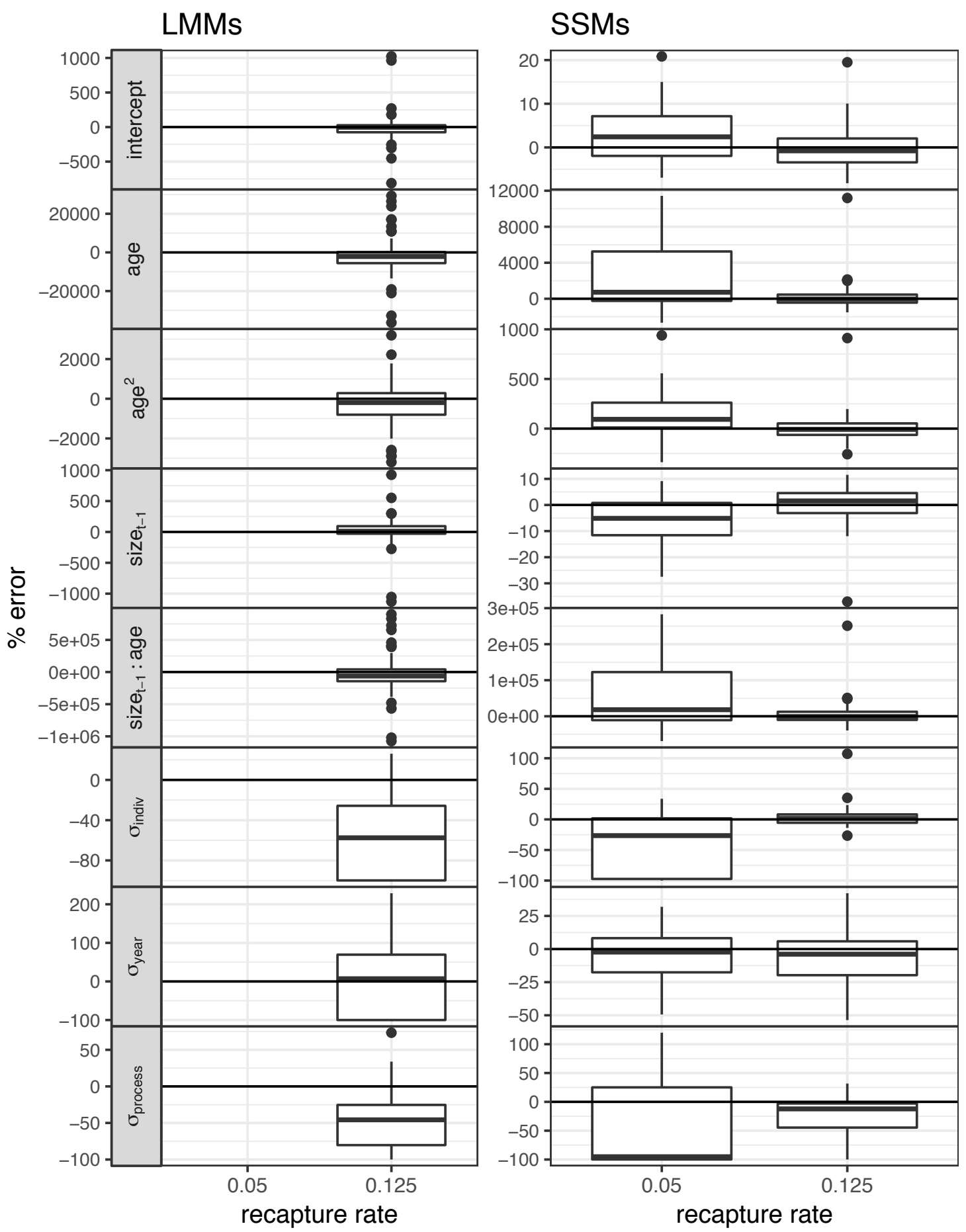

Figure S3-2 Error relative to true value. The left and right column represent linear mixed models (LMMs) and state-space models (SSMs) respectively fit to 100 simulated data sets. Data sets were simulated with recapture rates of 0.05 and 0.125 (i.e., $95 \%$ and $87.5 \%$ of repeated measures missing) which are represented by the x-axis. Each row of panels represents a different parameter of the model. Horisontal lines represent $0 \%$ error of the estimate relative to the true parameter used to simulate the growth data. Estimates are summarized by Tukey style boxplots in which boxes encompass the 25th to the 75 th quantile with a thicker line at the median, whiskers extend from the box up to 1.5 times the inner quartile range (truncated to the location of data), and points represent outliers. 
Table S3-1 The sheep's combinations of recap and sigma_obs. This table summarizes a subset of the simulated data that should be the most similar to the real sheep growth data. The average of estimates from 100 simulated data is presented.

\begin{tabular}{llrr}
\hline model & parameter & \% error & error \\
\hline LMM & intercept & 1 & 0.018 \\
LMM & age & -3116 & -0.119 \\
LMM & age^2 & -113 & 0.001 \\
LMM & prevsize & 1 & 0.006 \\
LMM & prevsize:age & -76398 & 0.035 \\
LMM & sigma[indiv] & -58 & -0.028 \\
LMM & sigma[year] & 1 & 0.000 \\
LMM & sigma[process] & 57 & 0.025 \\
SSM & intercept & 0 & -0.001 \\
SSM & age & 34 & 0.001 \\
SSM & age^2 & 2 & 0.000 \\
SSM & prevsize & 0 & 0.000 \\
SSM & prevsize:age & 827 & 0.000 \\
SSM & sigma[indiv] & 0 & 0.000 \\
SSM & sigma[year] & -3 & -0.001 \\
SSM & sigma[process] & -1 & 0.000 \\
\hline
\end{tabular}




\title{
Estimation of individual growth trajectories when repeated measures are missing
}

\author{
Mollie E. Brooks ${ }^{1,2, *}$, Christopher Clements ${ }^{1}$, Josephine Pemberton ${ }^{3}$, and Arpat Ozgul ${ }^{1}$
}

${ }^{1}$ Department of Evolutionary Biology and Environmental Studies, University of Zurich, Winterthurerstrasse 190, 8057 Zurich, Switzerland ${ }^{2}$ National Institute of Aquatic Resources, Technical University of Denmark,

Charlottenlund Slot, 2920 Charlottenlund, Denmark ${ }^{3}$ Institute of Evolutionary Biology, University of

Edinburgh, Edinburgh EH9 3JT, United Kingdom

\section{Online Appendix S4- Model selection of random effects}

In this appendix, we present the results of model selection on the structure of the random effects. As described in the main text, we first chose the age effect and then given that age effect, we consider other random effects structures. For all these models, observation error sigma_obs was held as constant based on the independent estimation described in the main text; that's why its estimated standard error is 0. For all these models, masses in the first August of life had the same estimated mean for all individuals (i.e., ignoring covariates). In the model selection table, we only present models that had less than 2 deltaAIC compared to the best model within a given class of models (i.e., given the same form of age and random effects).

For conciseness, we use the following abbreviations for the coefficients. They are described in more detail in the main text.

\begin{tabular}{ll}
\hline abreviation & meaning \\
\hline a & age \\
sta & stage: lamb ( $<1$ year), yearling, prime-aged adult $(2$ to 6 years $)$, and senescent ( $>6$ years $)$ \\
fa & factor of age (one level for each age, except $>12$ which is combined) \\
lamb & lamb status \\
rep & reproducive status \\
pop & population density \\
nao & winter NAO \\
i & individual \\
y & year \\
c & birth cohort \\
\hline
\end{tabular}

To concisely describe the random effects (RE) of each model here, we use the same notation as used by lme4.

\begin{tabular}{lllr}
\hline formulaX & formulaM & RE & deltaAIC \\
\hline$\sim \mathrm{a}+\mathrm{I}\left(\mathrm{a}^{\wedge} 2\right)+$ lamb+ rep+ pop+nao +lamb:pop & $\sim$ lamb+a+nao & $(1+\mathrm{a} \mid \mathrm{i})+(1 \mid \mathrm{y})+(1 \mid \mathrm{c})$ & 0.000 \\
$\sim \mathrm{a}+\mathrm{I}\left(\mathrm{a}^{\wedge} 2\right)+$ lamb+ rep+ pop+nao+ a:nao +lamb:pop & $\sim$ lamb+a & $(1+\mathrm{a} \mid \mathrm{i})+(1 \mid \mathrm{y})+(1 \mid \mathrm{c})$ & 0.880 \\
$\sim \mathrm{a}+\mathrm{I}\left(\mathrm{a}^{\wedge} 2\right)+$ lamb+ rep+ pop+nao + a:rep +lamb:pop & $\sim$ lamb+a+nao & $(1+\mathrm{a} \mid \mathrm{i})+(1 \mid \mathrm{y})+(1 \mid \mathrm{c})$ & 0.969 \\
$\sim \mathrm{a}+\mathrm{I}\left(\mathrm{a}^{\wedge} 2\right)+$ lamb+ rep+ pop+nao+lamb:nao +lamb:pop & $\sim$ lamb+a+nao & $(1+\mathrm{a} \mid \mathrm{i})+(1 \mid \mathrm{y})+(1 \mid \mathrm{c})$ & 0.973 \\
$\sim \mathrm{a}+\mathrm{I}\left(\mathrm{a}^{\wedge} 2\right)+$ lamb+ rep+ pop+nao + lamb:rep +lamb:pop & $\sim$ lamb+a+nao & $(1+\mathrm{a} \mid \mathrm{i})+(1 \mid \mathrm{y})+(1 \mid \mathrm{c})$ & 1.361 \\
$\sim \mathrm{a}+\mathrm{I}\left(\mathrm{a}^{\wedge} 2\right)+$ lamb+ rep+ pop+nao +lamb:pop+lamb:pop:nao & $\sim$ lamb+a+nao & $(1+\mathrm{a} \mid \mathrm{i})+(1 \mid \mathrm{y})+(1 \mid \mathrm{c})$ & 1.609 \\
$\sim \mathrm{a}+\mathrm{I}\left(\mathrm{a}^{\wedge} 2\right)+$ lamb+ rep+ pop+nao+ a:nao +lamb:pop & $\sim$ lamb+a+nao & $(1+\mathrm{a} \mid \mathrm{i})+(1 \mid \mathrm{y})+(1 \mid \mathrm{c})$ & 1.676 \\
$\sim \mathrm{a}+\mathrm{I}\left(\mathrm{a}^{\wedge} 2\right)+$ lamb+ rep+ pop+nao + a:rep+ a:nao +lamb:pop & $\sim$ lamb+a & $(1+\mathrm{a} \mid \mathrm{i})+(1 \mid \mathrm{y})+(1 \mid \mathrm{c})$ & 1.952 \\
$\sim \mathrm{a}+\mathrm{I}\left(\mathrm{a}^{\wedge} 2\right)+$ lamb+ rep+ pop+nao + a:rep +lamb:pop & $\sim$ lamb+a+nao & $(1 \mid \mathrm{i})+(1 \mid \mathrm{y})+(1 \mid \mathrm{c})$ & 34.251 \\
$\sim \mathrm{a}+\mathrm{I}\left(\mathrm{a}^{\wedge} 2\right)+$ lamb+ rep+ pop+nao + a:rep+ a:nao +lamb:pop & $\sim$ lamb+a & $(1 \mid \mathrm{i})+(1 \mid \mathrm{y})+(1 \mid \mathrm{c})$ & 35.290 \\
& & &
\end{tabular}

*Corresponding author. email MollieEBrooks@gmail.com 


\begin{tabular}{|c|c|c|c|}
\hline formulaX & formulaM & $\mathrm{RE}$ & deltaAIC \\
\hline$\sim \mathrm{a}+\mathrm{I}\left(\mathrm{a}^{\wedge} 2\right)+$ lamb+ rep+ pop+nao + a:rep+lamb:nao +lamb:pop & $\sim \operatorname{lamb}+\mathrm{a}+\mathrm{nao}$ & $(1 \mid \mathrm{i})+(1 \mid \mathrm{y})+(1 \mid \mathrm{c})$ & 35.874 \\
\hline$\sim a+I\left(a^{\wedge} 2\right)+$ lamb+rep+ pop+nao + a:rep+a:nao +lamb:pop & $\sim \operatorname{lamb}+\mathrm{a}+$ nao & $(1 \mid \mathrm{i})+(1 \mid \mathrm{y})+(1 \mid \mathrm{c})$ & 35.882 \\
\hline$\sim a+I\left(a^{\wedge} 2\right)+$ lamb+ rep+ pop+nao + a:rep +lamb:pop+lamb:pop:nao & $\sim \operatorname{lamb}+\mathrm{a}+$ nao & $(1 \mid \mathrm{i})+(1 \mid \mathrm{y})+(1 \mid \mathrm{c})$ & 35.923 \\
\hline$\sim a+\mathrm{I}\left(\mathrm{a}^{\wedge} 2\right)+$ lamb+ rep+ pop+nao + a:rep + lamb:rep +lamb:pop & $\sim \operatorname{lamb}+\mathrm{a}+\mathrm{nao}$ & $(1 \mid \mathrm{i})+(1 \mid y)+(1 \mid c)$ & 36.246 \\
\hline$\sim a+\mathrm{I}\left(\mathrm{a}^{\wedge} 2\right)+$ lamb+rep+ pop+nao + a:rep +lamb:pop & $\sim \operatorname{lamb}+\mathrm{a}+$ nao & $(1 \mid \mathrm{i})+(1 \mid y)$ & 37.695 \\
\hline$\sim a+I(a \wedge 2)+$ lamb+rep+ pop+nao + a:rep+a:nao +lamb:pop & $\sim \operatorname{lamb}+\mathrm{a}$ & $(1 \mid \mathrm{i})+(1 \mid y)$ & 38.679 \\
\hline$\sim a+\mathrm{I}\left(\mathrm{a}^{\wedge} 2\right)+$ lamb+rep+ pop+nao + a:rep +lamb:pop+lamb:pop:nao & $\sim \operatorname{lamb}+\mathrm{a}+\mathrm{nao}$ & $(1 \mid \mathrm{i})+(1 \mid y)$ & 39.050 \\
\hline$\sim a+\mathrm{I}\left(\mathrm{a}^{\wedge} 2\right)+$ lamb+ rep+ pop+nao + a:rep+lamb:nao +lamb:pop & $\sim \operatorname{lamb}+\mathrm{a}+\mathrm{nao}$ & $(1 \mid \mathrm{i})+(1 \mid y)$ & 39.356 \\
\hline$\sim a+I\left(a^{\wedge} 2\right)+$ lamb+ rep+ pop+nao + a:rep+ a:nao +lamb:pop & $\sim \operatorname{lamb}+\mathrm{a}+$ nao & $(1 \mid \mathrm{i})+(1 \mid \mathrm{y})$ & 39.372 \\
\hline$\sim \mathrm{a}+\mathrm{I}\left(\mathrm{a}^{\wedge} 2\right)+$ lamb+ rep+ pop+nao + a:rep + lamb:rep +lamb:pop & $\sim \operatorname{lamb}+\mathrm{a}+\mathrm{nao}$ & $(1 \mid \mathrm{i})+(1 \mid \mathrm{y})$ & 39.692 \\
\hline
\end{tabular}

We can use summary ( $)$ to check the estimates of the top model to make sure that they are valid.

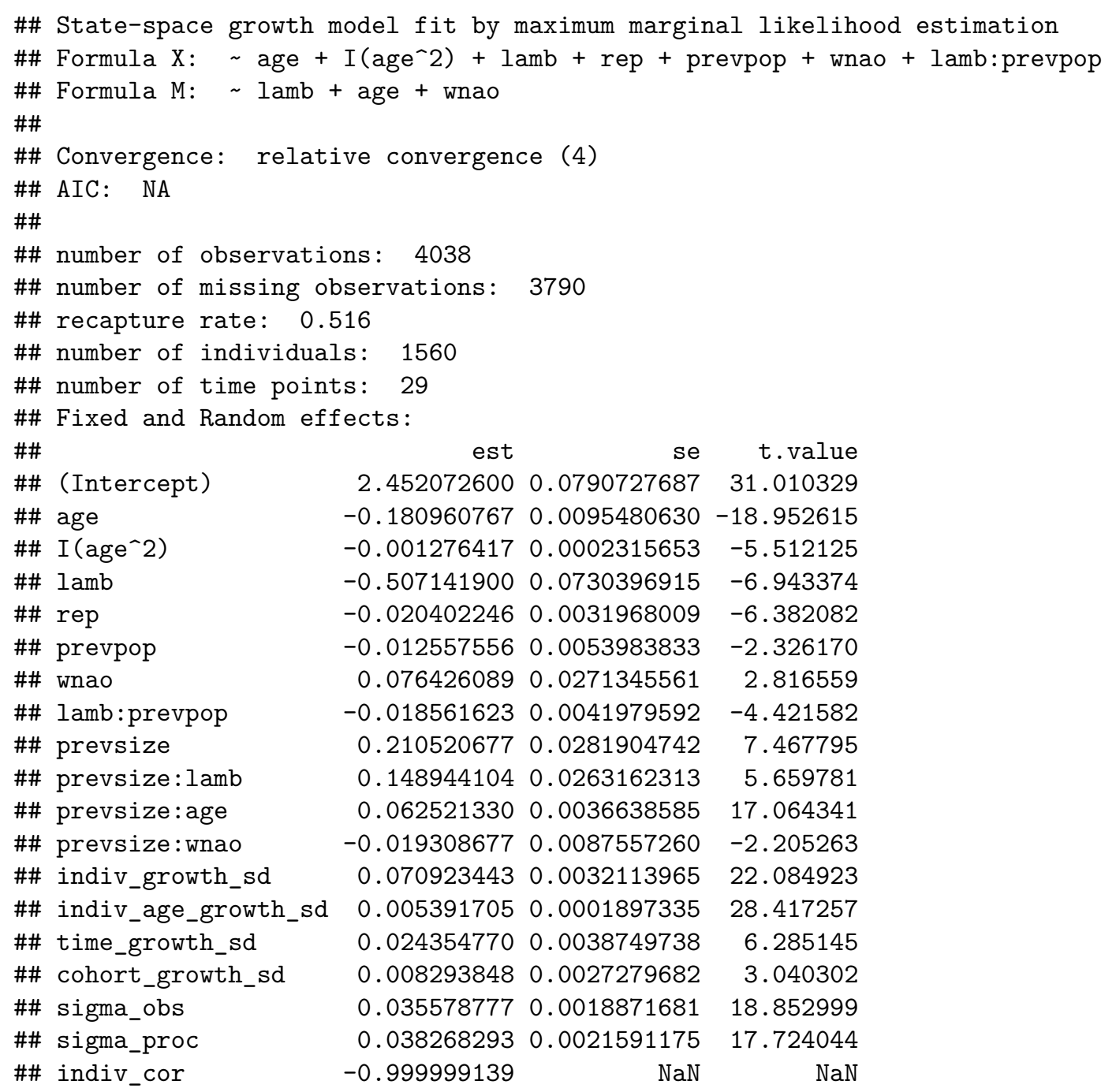

Having a correlation of -0.999999 indicates that the model is degenerate (Bates et al. 2015b). 


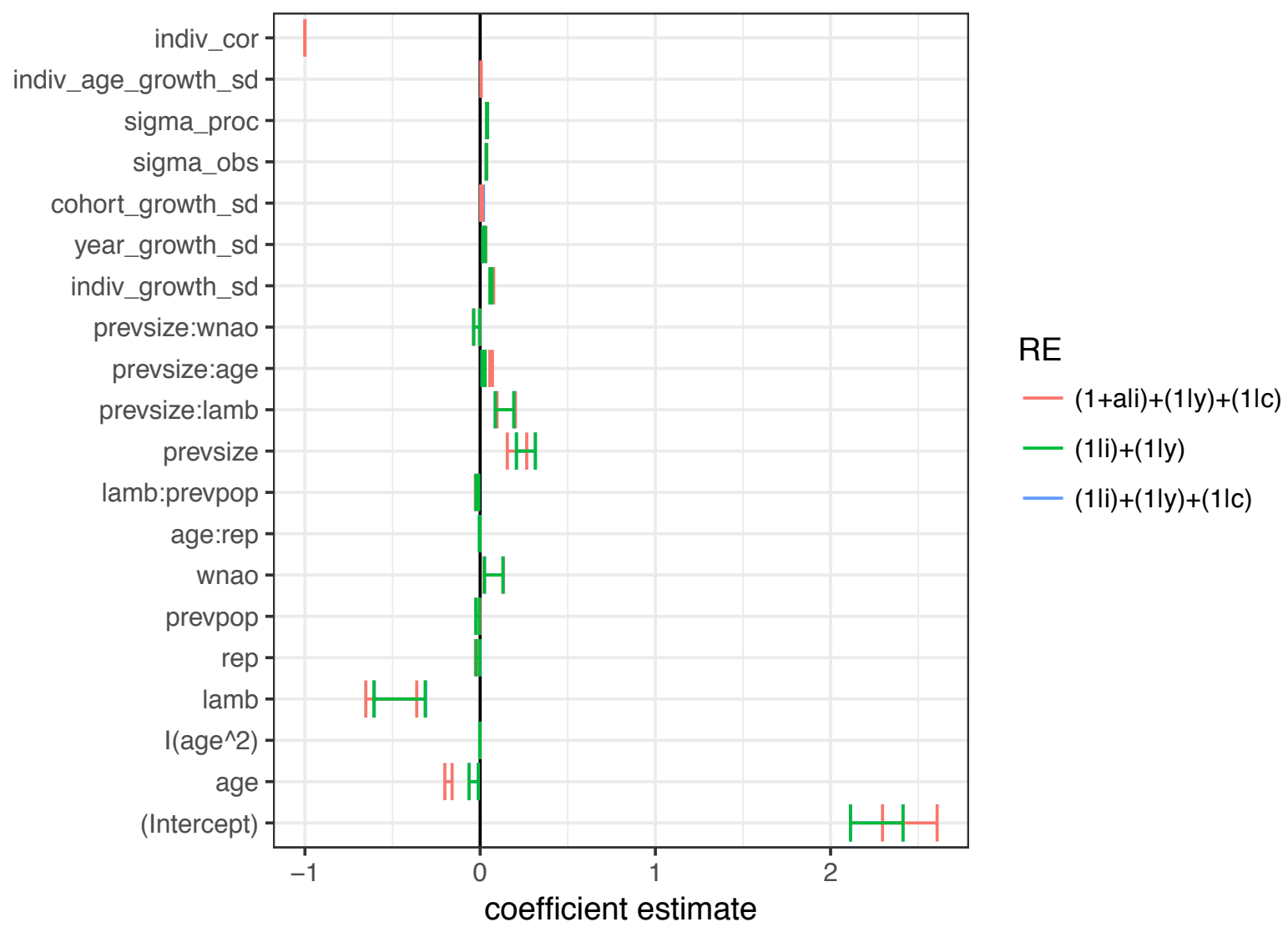

The coefficient estimates are fairly consistent regardless of the random effects structure. 